\title{
Periodic ordering of clusters in a one- dimensional lattice model
}

Cite as: J. Chem. Phys. 138, 144903 (2013); https://doi.org/10.1063/1.4799264

Submitted: 23 January 2013. Accepted: 19 March 2013. Published Online: 11 April 2013

J. Pękalski, A. Ciach, and N. G. Almarza

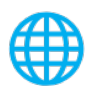

\section{ARTICLES YOU MAY BE INTERESTED IN}

Periodic ordering of clusters and stripes in a two-dimensional lattice model. I. Ground state, mean-field phase diagram and structure of the disordered phases

The Journal of Chemical Physics 140, 114701 (2014); https://doi.org/10.1063/1.4868001

Cluster formation in fluids with competing short-range and long-range interactions

The Journal of Chemical Physics 140, 124508 (2014); https://doi.org/10.1063/1.4869109

Periodic ordering of clusters and stripes in a two-dimensional lattice model. II. Results of Monte Carlo simulation

The Journal of Chemical Physics 140, 164708 (2014); https://doi.org/10.1063/1.4871901

\section{Challenge us.}

What are your needs for periodic signal detection?

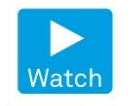

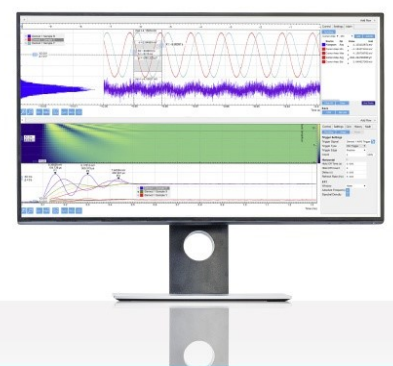

Zurich

Instruments 


\title{
Periodic ordering of clusters in a one-dimensional lattice model
}

\author{
J. Pẹkalski, ${ }^{1}$ A. Ciach, ${ }^{1}$ and N. G. Almarza ${ }^{2}$ \\ ${ }^{1}$ Institute of Physical Chemistry, Polish Academy of Sciences, 01-224 Warszawa, Poland \\ ${ }^{2}$ Instituto de Química Física Rocasolano, CSIC, Serrano 119, E-28006 Madrid, Spain
}

(Received 23 January 2013; accepted 19 March 2013; published online 11 April 2013)

\begin{abstract}
A generic lattice model for systems containing particles interacting with short-range attraction longrange repulsion (SALR) potential that can be solved exactly in one dimension is introduced. We assume attraction $J_{1}$ between the first neighbors and repulsion $J_{2}$ between the third neighbors. The ground state of the model shows existence of two homogeneous phases (gas and liquid) for $J_{2} / J_{1}$ $<1 / 3$. In addition to the homogeneous phases, the third phase with periodically distributed clusters appears for $J_{2} / J_{1}>1 / 3$. Phase diagrams obtained in the self-consistent mean-field approximation for a range of values of $J_{2} / J_{1}$ show very rich behavior, including reentrant melting, and coexistence of two periodic phases (one with strong and the other one with weak order) terminated at a critical point. We present exact solutions for the equation of state as well as for the correlation function for characteristic values of $J_{2} / J_{1}$. Based on the exact results, for $J_{2} / J_{1}>1 / 3$ we predict pseudo-phase transitions to the ordered cluster phase indicated by a rapid change of density for a very narrow range of pressure, and by a very large correlation length for thermodynamic states where the periodic phase is stable in mean field. For $1 / 9<J_{2} / J_{1}<1 / 3$ the correlation function decays monotonically below certain temperature, whereas above this temperature exponentially damped oscillatory behavior is obtained. Thus, even though macroscopic phase separation is energetically favored and appears for weak repulsion at $T=0$, local spatial inhomogeneities appear for finite $T$. Monte Carlo simulations in canonical ensemble show that specific heat has a maximum for low density $\rho$ that we associate with formation of living clusters, and if the repulsion is strong, another maximum for $\rho=1 / 2$. (c) 2013 American Institute of Physics. [http://dx.doi.org/10.1063/1.4799264]
\end{abstract}

\section{INTRODUCTION}

Most of biologically relevant macromolecules, or particles in soft-matter systems are charged and repel each other with screened electrostatic forces. ${ }^{1-4}$ On the other hand, complex solvents in biological or soft-matter systems may induce effective attraction between the macromolecules. Important examples of the effective attraction include depletion forces resulting from the presence of small objects such as nonadsorbing polymers, ${ }^{4-6}$ solvophobic attraction, ${ }^{7,8}$ or thermodynamic Casimir forces ${ }^{9-12}$ resulting from critical adsorption near a critical demixing point in a binary (or multicomponent) solvent. The sum of all interactions has often the form of short-range attraction long-range repulsion (SALR) potential. ${ }^{13-27}$

Despite of the importance of the SALR potential for biological and soft-matter systems only preliminary and incomplete results for phase diagrams have been obtained so far. ${ }^{14-26}$ It is clear that the phase diagrams depend on the strength of the repulsion, and for strong enough repulsion can be completely different than in simple fluids. In the latter systems the phase behavior is determined by the competition between the entropy favoring the disordered phase and the energy favoring formation of a spherical droplet of the dense phase. Volume fraction of the particles determines only the size of this droplet. In the case of the SALR potential there is additional competition between the energy and the chemical potential (or the volume fraction) of particles. The SALR potential favors formation of spherical clusters whose size is determined by the range of the attraction, and the distance between the clusters is determined by the range of the repulsion. The structure minimizing the energy is possible only for sufficiently small volume fraction, however. The increase of the chemical potential leads to transitions from spherical to cylindrical clusters, next to a network, then to layers of particles, and finally to inverse structures (occupied regions replaced by voids). ${ }^{4,20,21,23,24,26}$ Regions of stability of the disordered fluid and different ordered phases, as well as location and order of phase-transitions between them for various shapes of the SALR potential are only partially known.

The difficulty in determination of phase diagrams in experimental studies results from the presence of many metastable phases and large characteristic time scales, by which instantaneous rather than average states have been observed. ${ }^{3,4}$ The question of the average structure in thermodynamic equilibrium may be clarified in future confocal microscopy dynamical studies. In simulations it is much more difficult to study inhomogeneous structures with some kind of periodic ordering and moreover the results are restricted to the chosen form of interactions. ${ }^{15-17,23,26,27}$ In theoretical studies the results relay on approximations, usually of the mean-field (MF) type. ${ }^{14,18-25}$ Validity of MF for the SALR type potentials was only partially verified, ${ }^{20,23}$ and it is not clear which predictions of the MF approximation and to what extent are valid on a qualitative or on a semiquantitative level. Preliminary results within field-theoretic approach show that fluctuations may lead to substantial modification of the equation of $\operatorname{state}^{28}$ and to a fluctuation-induced first 
order phase transition, ${ }^{20,29}$ as in magnetic systems with competing interactions. ${ }^{30,31}$ We should also mention that the accurate liquid theories such as self-consistent Ornstein-Zernike approximation (SCOZA) $)^{32}$ are unfortunately limited to uniform phases, and only a lack of solutions is an indicator of possible phases with periodic ordering on the mesoscopic length scale. ${ }^{14,18,19}$ Further studies including comparison between MF type results and simulations, as in Ref. 23 are necessary. It would be also desirable to introduce a simple model that could be solved exactly. Exact results could serve for testing general theoretical predictions and for verification of the range of validity of approximate theories. No simple model that could play the same role as the two-dimensional Ising model played for simple systems was proposed so far.

An exactly solvable two-dimensional model for the SALR potential cannot be proposed at the moment. To fill this gap at least partially, we introduce in this work a onedimensional (1D) lattice model with attraction $J_{1}$ between the first and repulsion $J_{2}$ between the third neighbors. We assume that the lattice constant is equal to the size $\sigma$ of the particles. With this form of the potential the energy is minimized when clusters of the size $3 \sigma$ are formed. This size is intermediate between very small (dimers) or quite large linear extensions of the aggregates, $2 \sigma$ or $11 \sigma$, studied in Ref. 26 or Refs. 15, 17, 21, and 23. Exact solutions can be obtained for the equation of state (EOS) as well as for the density and the correlation function for the whole range of $J_{2} / J_{1}$ in the grand canonical ensemble. We shall calculate and analyze the EOS and the correlation function for broad ranges of temperature $T$ and the chemical potential $\mu$ for strong, medium and weak repulsion.

The specific heat is measured for fixed number of particles. Fixed number of particles imposes a global constraint on the microscopic states, and analytical calculations in the canonical ensemble become difficult even in the case of the lattice gas equivalent to the Ising model. ${ }^{33}$ For this reason in order to obtain information on thermal properties of systems with competing interactions we perform Monte Carlo (MC) simulations in the canonical ensemble. The specific heat $c_{v}$ is often computed in simulations in order to determine phase transitions. ${ }^{17}$ However, large energy fluctuations may be associated with formation of living (or dynamic) clusters, especially when the clusters easily form or dissociate. In order to avoid misinterpretation of the simulation results for higher dimensions, it is important to distinguish the properties of the $c_{v}$ that are associated with phase transitions from those associated with formation of living clusters. For this reason we shall calculate $c_{v}$ for the 1D system, where no true phase transitions occur, but the distribution of particles can be strongly inhomogeneous. We calculate the specific heat for several temperatures as function of density for strong and for weak repulsion.

There are no phase transitions in 1D models with finite range of interactions, but pseudo-phase transitions may exist for low temperatures. It is of interest to compare thermodynamics and structure of the disordered phase in simple fluid and in the SALR-potential systems. The exact results can give interesting information about pretransitional ordering (including cluster formation and distribution in space) and how this ordering is reflected in measurable quantities. Effects of the precursors of the ordered structures on structure, compressibility, and specific heat were already studied in approximate theories and in simulations. ${ }^{14-19,25}$ Based on the exact results for the correlation function and for the EOS, we can verify if the repulsion can lead to significantly increased compressibility and to oscillatory decay of correlations on a mesoscopic length scale, as found in Refs. 18-23.

In MF phase transitions are present even in one dimension, therefore MF in 1D systems can shed light on some general features of phase diagrams in two or three dimensions. The main difference between our results and phase diagrams in $2 \mathrm{D}$ or 3D systems should follow from formation of ordered structures with periodic distribution of particles in 1, 2, and 3 dimensions. Finally, it is interesting to compare the exact and MF results in order to better interpret the latter.

The one-dimensional model considered here, apart from yielding general information on periodic ordering in the SALR-potential systems, describes several physical systems that are interesting by their own. In the first place it can represent charged particles in a presence of depletant at a threephase coexistence line, or adsorbed at nanotubes or microtubules. Another example is a linear backbone polymer with monomers containing sites binding particles or ions that attract or repel each other when bound to first or third neighbors on the backbone, respectively. Our model can answer the question of spontaneous formation of ordered periodic patterns on linear substrates.

Let us finally mention that competing interactions are present not only in soft-matter and biological systems. Lattice models with competing interactions were introduced before for quite different systems. For example, various models with competing tendencies can describe water anomalies. ${ }^{34-38}$ In particular, competing short-range repulsion with longer range attraction can lead to density anomaly. ${ }^{37,38}$ Spatial inhomogeneities are expected when the range of repulsion is larger than the range of attraction. Alternating dense and dilute regions studied in this work are similar to periodic distribution of up and down spins in magnetic systems. Periodic distribution of up and down spins was obtained previously in the axial (or anisotropic) next nearest neighbor Ising (ANNNI) model with nearest-neighbor ferromagnetic and next-nearestneighbor antiferromagnetic interaction in one direction, with ferromagnetic coupling in the remaining directions. ${ }^{31,39-41}$ Very rich phase behavior, including periodic phases with periods incommensurate with the lattice, was obtained for various ratios of the ferromagnetic and antiferromagnetic couplings and the exact expression for the correlation function was found. The studies, however, did not focus on the role of the external magnetic field that in the fluid version of the lattice models corresponds to the chemical potential $\mu$. In the context of fluids the chemical potential plays a crucial role and allows to obtain the EOS and structure for dense and dilute fluids.

In Sec. II the model is introduced and the ground state is determined and discussed. Section III is devoted to the MF approximation. Exact results and MC simulations are described in Secs. IV and V, respectively. Section VI contains a short summary and discussion. 


\section{THE MODEL AND ITS GROUND STATE}

\section{A. The model}

We consider an open system in equilibrium with a reservoir with temperature $T$ and chemical potential $\mu_{p}$. The interaction $h$ between the particles and the nanotubes, microtubules, or binding sites plays analogous role as the chemical potential, and we introduce $\mu=\mu_{p}+h$. The particles can occupy lattice sites labeled by $x$ taking integer values, $1 \leq x \leq L$ and we assume periodic boundary conditions (PBC), i.e., $L+1 \equiv 1,0 \equiv L$. Each microstate is described by $\{\hat{\rho}(x)\} \equiv(\hat{\rho}(1), \ldots, \hat{\rho}(L))$, where the occupancy operator $\hat{\rho}(x)=1$ or $\hat{\rho}(x)=0$ when the site $x$ is occupied or empty, respectively. The probability of the microstate $\{\hat{\rho}(x)\}$ is given by

$$
p[\{\hat{\rho}(x)\}]=\frac{e^{-\beta H[\{\hat{\rho}(x)\}]}}{\Xi},
$$

where $\Xi$ is the normalization constant, and $\beta=\left(k_{B} T\right)^{-1}$ with $k_{B}$ denoting the Boltzmann constant. We have introduced for convenience the thermodynamic Hamiltonian containing the energy and the chemical potential term:

$$
H[\{\hat{\rho}\}]=\frac{1}{2} \sum_{x=1}^{L} \sum_{x^{\prime}=1}^{L} \hat{\rho}(x) V\left(x-x^{\prime}\right) \hat{\rho}\left(x^{\prime}\right)-\mu \sum_{x=1}^{L} \hat{\rho}(x),
$$

where the interaction potential is

$$
\begin{aligned}
V(\Delta x)= & -J_{1}\left(\delta^{K r}(\Delta x+1)+\delta^{K r}(\Delta x-1)\right) \\
& +J_{2}\left(\delta^{K r}(\Delta x+3)+\delta^{K r}(\Delta x-3)\right) .
\end{aligned}
$$

In the above $\delta^{K r}$ is the Kronecker delta. The Hamiltonian (2) can be rewritten in terms of the "unoccupancy" operator $\hat{v}(x)=1-\hat{\rho}(x)(\hat{v}(x)=1,0$ for an empty and full site $x$, respectively):

$$
\begin{aligned}
H[\{\hat{v}\}]= & \frac{1}{2} \sum_{x=1}^{L} \sum_{x^{\prime}=1}^{L} \hat{v}(x) V\left(x-x^{\prime}\right) \hat{v}\left(x^{\prime}\right) \\
& +\left(\mu-V_{0}\right) \sum_{x=1}^{L} \hat{v}(x)+L\left(\frac{V_{0}}{2}-\mu\right),
\end{aligned}
$$

where $V_{0}=\sum_{x} V(x)=2\left(J_{2}-J_{1}\right)$. Note that Eq. (2) in terms of $\hat{\rho}$ and Eq. (4) in terms of $\hat{v}$ have the same form for $\mu=V_{0} / 2$. Moreover, the probability of the microstate $\{\hat{\rho}(x)\}$ for $\mu=V_{0} / 2-\Delta \mu$ is the same as the probability of the "negative" of this microstate, $\{1-\hat{\rho}(x)\}$, for $\mu=V_{0} / 2+\Delta \mu$. Because of the above particle-hole symmetry the phase diagrams must be symmetric with respect to the symmetry axis $\mu=J_{2}-J_{1}$.

We choose $J_{1}$ as the energy unit and introduce dimensionless variables for any quantity $X$ with dimension of energy as $X^{*}=X / J_{1}$, in particular

$$
T^{*}=k_{B} T / J_{1}, J^{*}=J_{2} / J_{1}, \mu^{*}=\mu / J_{1} .
$$

\section{B. The ground state}

The grand potential:

$$
\Omega=-p L=-k_{B} T \ln \Xi=U-T S-\mu N,
$$

where $p, U, S, N$ are pressure, internal energy, entropy, and average number of particles, respectively, reduces to the minimum of $H[\{\hat{\rho}(x)\}]$ for $T=0$ and fixed $L$. In the case of periodic phases the bulk properties must be determined for $L=$ $l n$, where $l$ is the period of density oscillations and $n$ is integer. We consider $\omega^{*}=-p^{*}=H^{*}[\{\hat{\rho}(x)\}] /(\ln )$ for two homogeneous phases, one with all sites empty (gas) and the other one with all sites occupied (liquid) and for a periodic phase where three occupied neighboring sites are followed by $l-3$ empty sites with $l \geq 6$. For these phases we have

$$
\omega^{*}=\left\{\begin{array}{ll}
0 & \text { empty lattice (gas) } \\
-\frac{2+3 \mu^{*}}{l} & \text { periodic, } l \geq 6 \\
J^{*}-1-\mu^{*} & \text { full occupancy (liquid) }
\end{array} .\right.
$$

Two phases can coexist for thermodynamic states such that $\omega^{*}$ in these phases takes the same value. The $\left(J^{*}, \mu^{*}\right)$ phase diagram for $T^{*}=0$ is shown in Fig. 1.

Note that for $\mu^{*}=-2 / 3$ the $\omega^{*}$ of the periodic phase is independent of $l$ if $l \geq 6$. This is because when in the empty lattice 3 neighboring cells become occupied, the associated change of $H^{*}$ is $-2-3 \mu^{*}$. Because the interaction range is 3 , for $\mu^{*}=-2 / 3$ a triple of occupied cells can be separated from another triple of occupied cells by $l-3$ empty cells for any $l \geq 6$. Such a state can be interpreted as a cluster fluid that can be stable, however, for a single value of the chemical potential, $\mu^{*}=-2 / 3$. When $2+3 \mu^{*}>0$, the lowest value of $\omega^{*}$ corresponds to $l=6$, and the periodic phase with period 6 is stable. The gas and periodic phases coexist for $\mu^{*}=-2 / 3$. Because of this coexistence, arbitrary separation between the clusters, whose number is also arbitrary (but smaller than $L / 6$ ), can be interpreted as arbitrarily small droplets (larger than 6 in the case of the periodic phase) of these phases. As a result, an arbitrary number of interfaces can be formed. This is possible when the surface tension between the gas and the periodic phases vanishes.

Similarly, creation of a triple of empty sites in the fully occupied lattice leads to the change of $H^{*}$ which is $\Delta H^{*}$ $=-6 J^{*}+4+3 \mu^{*}$. At the coexistence between the fully

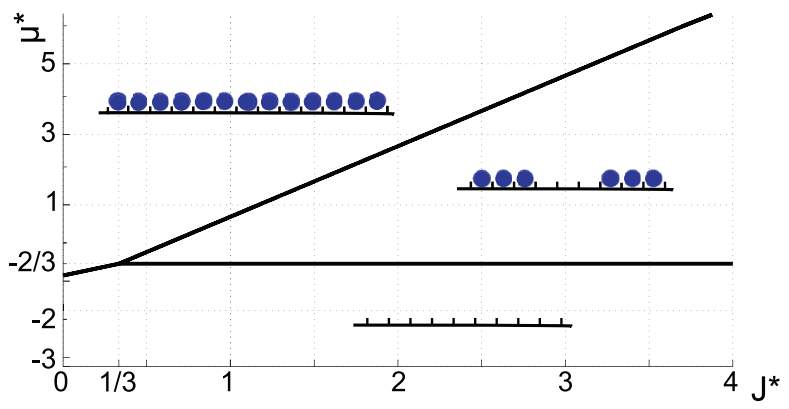

FIG. 1. Ground state of the considered model. The repulsion to attraction ratio $J^{*}$ and the chemical potential $\mu^{*}$ are dimensionless (see (5)). The coexistence lines are $\mu_{\text {gas-cond }}^{*}=-1+J^{*}, \mu_{\text {gas-per }}^{*}=-2 / 3$, and $\mu_{\text {cond-per }}^{*}$ $=-4 / 3+2 J^{*}$. Schematic illustration of the three phases is shown in the insets inside the region of stability of each phase. 
occupied lattice and the periodic phase $6 J^{*}-4-3 \mu^{*}=$ 0 , hence the separation between the three empty neighboring cells (bubbles) can be arbitrary (but $\geq 3$ ). Again, such a state can be interpreted as a fluid of bubbles, or as a coexistence between the liquid and periodic phases in the case of vanishing surface tension. Note the similarity between this property of our model and the very low surface tension between wateror oil-rich phases and microemulsion. At $T^{*}=0$ formation of the microemulsion is associated with vanishing surface tension in the lattice model for the water-oil-surfactant mixture. ${ }^{42}$

\section{MF APPROXIMATION}

\section{A. Short background}

In the self-consistent MF approximation the Hamiltonian (2) is approximated by

$$
H^{M F}[\{\hat{\rho}(x)\}]=\sum_{x}\left[-(h(x)+\mu) \hat{\rho}(x)+\frac{1}{2} h(x) \bar{\rho}(x)\right],
$$

where the mean-field acting on the site $x$ is

$$
h(x)=-\sum_{x^{\prime}} V\left(x-x^{\prime}\right) \bar{\rho}\left(x^{\prime}\right)
$$

and the MF average density satisfies the self-consistent equation:

$$
\bar{\rho}(x)=\frac{e^{\beta(h(x)+\mu)}}{1+e^{\beta(h(x)+\mu)}} .
$$

The grand statistical sum:

$$
\Xi=\prod_{x}\left[e^{-\frac{\beta}{2} h(x) \bar{\rho}(x)}\left(1+e^{\beta(h(x)+\mu)}\right)\right]
$$

together with (9) after some algebra leads to the grand potential of the form: ${ }^{43}$

$$
\begin{aligned}
\Omega= & \sum_{x_{1}=1}^{L} \sum_{x_{2}=1}^{L}\left\{\frac{1}{2} \bar{\rho}\left(x_{1}\right) \bar{\rho}\left(x_{2}\right) V\left(x_{1}-x_{2}\right)\right. \\
& \left.+\delta^{K r}\left(x_{1}-x_{2}\right) f_{h}\left(\bar{\rho}\left(x_{1}\right)\right)\right\}-\mu \sum_{x=1}^{L} \bar{\rho}(x) .
\end{aligned}
$$

where in the lattice models

$$
f_{h}(\rho)=-k_{B} T s(\rho)=k_{B} T[\rho \ln (\rho)+(1-\rho) \ln (1-\rho)] .
$$

Local minima of (11) satisfy Eq. (9) (see Ref. 43).

Equation (9) can be solved by iterations for different initial conditions. Stability regions of different phases and firstorder transitions between them can be obtained by comparing $\omega=\Omega /(\ln )$ for different forms of $\bar{\rho}(x)$. In practice systems with the size $l$ and PBC represent one period of the phases with the period $l$, and we have considered $6 \leq l \leq 50$.

\section{B. Stability analysis}

Boundary of stability of the disordered phase can be found by analyzing the second derivative of $\beta \Omega$ with respect to the density profile $\bar{\rho}(x)$. The disordered fluid is stable as long as this derivative,

$$
C\left(x, x^{\prime}\right)=\frac{\partial \beta \Omega}{\partial \bar{\rho}(x) \partial \bar{\rho}\left(x^{\prime}\right)},
$$

is positive definite. The disordered phase is at the boundary of stability when the smallest eigenvalue of (13) vanishes for $\rho(x)=$ const. For interactions depending only on $x-x^{\prime}$ the quadratic part of $\beta \Omega$ (bilinear form) is diagonal in Fourier representation, and the eigenvalues of $C$ are given by

$$
\tilde{C}(k)=\beta^{*} \tilde{V}(k)+\frac{1}{\bar{\rho}(1-\bar{\rho})},
$$

where

$$
\tilde{V}(k)=\sum_{x} V^{*}(x) e^{i k x}
$$

In this model

$$
\tilde{V}(k)=-2 \cos k+2 J^{*} \cos 3 k .
$$

$\tilde{C}(k)$ assumes the smallest value $\tilde{C}\left(k_{b}\right)$ for given $T^{*}$ and $\mu^{*}$ for $k=k_{b}$ corresponding to the minimum of $\tilde{V}(k)$. We obtain

$$
k_{b}= \begin{cases}0 & \text { if } J^{*}<1 / 9, \\ \arccos \sqrt{\frac{1+3 J^{*}}{12 J^{*}}} & \text { otherwise, }\end{cases}
$$

and

$$
\tilde{V}\left(k_{b}\right)= \begin{cases}2\left(J^{*}-1\right) & \text { if } J^{*}<1 / 9, \\ -2 J^{*}\left(\frac{1+3 J^{*}}{3 J^{*}}\right)^{3 / 2} & \text { otherwise. }\end{cases}
$$

The boundary of stability of the disordered phase obtained from $\tilde{C}\left(k_{b}\right)=0$,

$$
k_{B} T^{*}=-\tilde{V}\left(k_{b}\right) \bar{\rho}_{0}\left(1-\bar{\rho}_{0}\right),
$$

represents the spinodal line of the gas-liquid separation when $k_{b}=0\left(J^{*}<1 / 9\right)$. The MF boundary of stability of the disordered phase with respect to density waves with finite wavelengths $2 \pi / k_{b}$ is termed $\lambda$-line in literature, ${ }^{19,23,44}$ to distinguish it from the spinodal line where the disordered phase is unstable with respect to phase separation. In this model the MF instability with respect to periodic ordering with the wavelength $2 \pi / k_{b}$ with $k_{b}>0$ occurs when $J^{*}>1 / 9$ (see Fig. 2).

It is interesting to find the $\lambda$-line and the spinodal in the $\left(\mu^{*}, T^{*}\right)$ phase space. From the form of the chemical potential

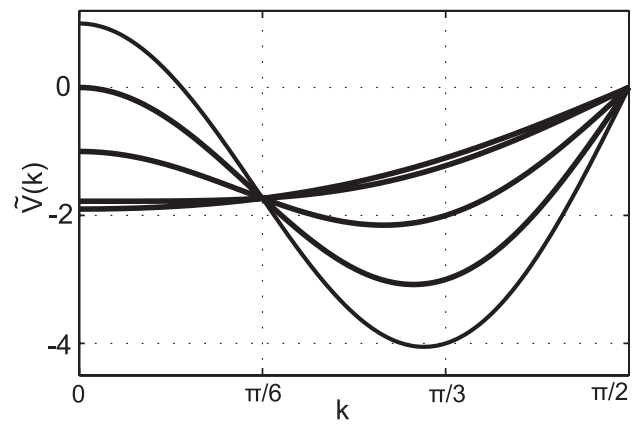

FIG. 2. $\tilde{V}(k)$ given by (16). $J^{*}=0.05,1 / 9,0.5,1,1.5$ from the bottom to the top line on the left. 


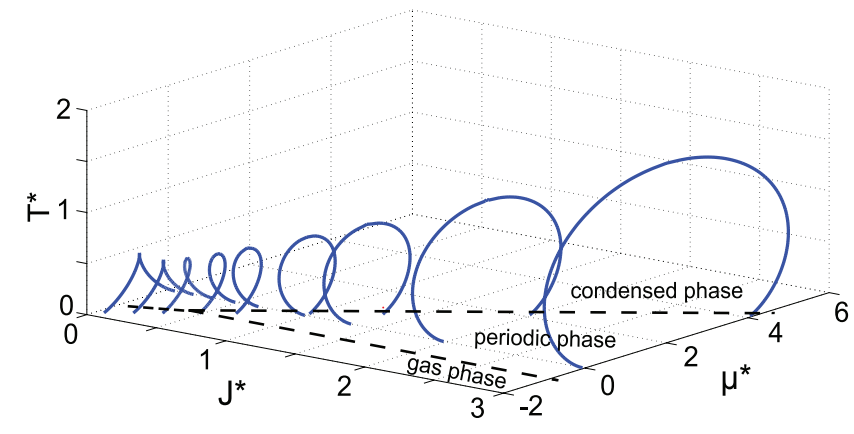

FIG. 3. Lines of instability (solid) of the homogeneous phase in the $\left(\mu^{*}, T^{*}\right)$ variables for a range of $J^{*}$. Similar behavior was obtained in Ref. 45. The coexistence lines at $T^{*}=0$ are shown as dashed lines.

for $\rho=$ const, $\mu^{*}=2\left(J^{*}-1\right) \rho+T^{*} \ln [\rho /(1-\rho)]$, we obtain the boundary of stability of the homogeneous phase

$\mu^{*}=\left(J^{*}-1\right)(1 \pm q)+T^{*} \ln \left(\frac{J^{*}}{2 T^{*}}\left(\frac{1+3 J^{*}}{3 J^{*}}\right)^{3 / 2}(1 \pm q)^{2}\right)$

where

$$
q=\sqrt{1+2 \frac{T^{*}}{J^{*}}\left(\frac{3 J^{*}}{1+3 J^{*}}\right)^{3 / 2}} .
$$

The shapes of the spinodal and $\lambda$-lines for various $J^{*}$ are shown in Fig. 3.

In the case of attraction dominated system $\left(J^{*}<1 / 9\right)$, the two branches of the spinodal line separate the region where $\Omega$ assumes two minima for two different constant densities (low$T^{*}$ side of the lines) from the region with one minimum on the high- $T^{*}$ side of the lines. This is usual behavior associated with the gas-liquid separation.

For $1 / 9<J^{*}<1$ the branches of the line of instability intersect and form a loop for high $T^{*}$. The homogeneous phase is unstable for any density inside the loop. Note that when the loop is present, then for decreasing $T^{*}$ or increasing $\mu^{*}$ there may exist a sequence of phases: disordered-periodicdisordered. Such a sequence agrees with observations of the reentrant melting. ${ }^{46,47}$ For the repulsion-dominated system $\left(J^{*}>1\right)$ only the loop is present (Fig. 3), and coexistence between homogeneous phases is not expected.

Our stability analysis is incomplete, because we did not study the boundary of stability of the periodic phase.

\section{MF phase diagrams}

The continuous transitions between the disordered and ordered phases coincide with the critical point or with the $\lambda$ line determined above for $J^{*}<1 / 9$ or $J^{*}>1 / 9$. The transitions become first order for $T^{*}<T_{t c p}^{*}$, where the tricritical point (TCP) is calculated analytically in the Appendix. The TCPs exist only for $1 / 9<J^{*}<1$. For $J^{*}=1 / 9$ the two TCP (one for $\rho<1 / 2$, the other one for $\rho>1 / 2$ ) merge into the critical point at $\rho=1 / 2$. For $J^{*}=1$ we obtain $T_{t c p}^{*}=0$. The locations of the first-order transition lines have been obtained by calculating the grand potential (11) for the average densities that are self-consistent solutions of Eq. (9). The method

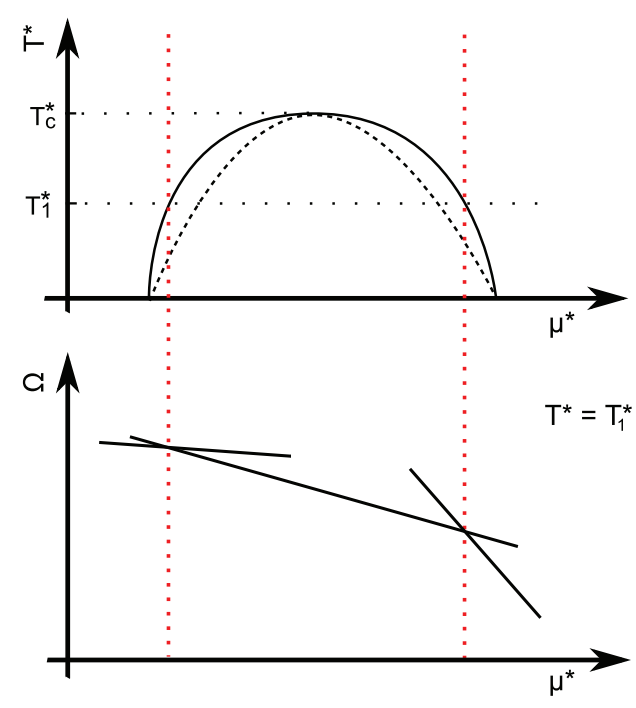

FIG. 4. Illustration of the method used for obtaining the phase coexistence. In the bottom panel the left, central, and right lines correspond to the gas, periodic, and disordered liquid phases, respectively.

of determining the transition lines is shown schematically in Fig. 4.

We obtain four qualitatively different phase diagrams for $J<1 / 9,1 / 9<J<1 / 3,1 / 3<J<1$, and $J>1$. In the first case standard gas-liquid separation occurs. For $1 / 9<J<1 / 3$ we find gas-liquid coexistence at low $T^{*}$, next a triple point where the two phases coexist with the periodic phase, and the first-order transition between the periodic phase and the fluid becomes continuous at the tricritical points. This type of phase diagram is shown in Fig. 5 for $J^{*}=1 / 4$. Similar phase diagram was obtained for very weak repulsion in Ref. 22, where only one-dimensional density oscillations were assumed in the Landau-type and density-functional theories. Moreover, when electrostatic repulsion is added to the Landau functional, similar phase diagram is obtained. ${ }^{48}$

For $1 / 3<J<1$ there is no coexistence between the gas and liquid phases. Instead, the gas-periodic phase transition, followed by the periodic phase-liquid transition occur for increasing chemical potential. The transitions are first order below and continuous above (in temperature) the tricritical points. The phase diagram for $J^{*}=1 / 3$ is shown in Fig. 6 . Similar phase behavior was obtained in Ref. 22 for mediumstrength repulsion.

Finally, for $J>1$ the tricritical points disappear and the transition between the disordered and periodic phases is continuous. This seems to be inconsistent with the presence of the first-order transition between the periodic and the fluid phases at $T^{*}=0$. This apparent inconsistency follows from the presence of two periodic phases for $J^{*}>1$. One of them is the same as the phase stable at $T^{*}=0$. It has large amplitude of density oscillations and the period $l=6$. The other phase appears inside the loop of the $\lambda$-line, has a period $2 \pi / k_{b}$ and small amplitude of density oscillations. The two periodic phases coexist along the line which is a continuation of the coexistence line between the large-amplitude periodic phase and the homogeneous fluid, above the temperature at which this transition and the low- $T^{*}$ branch of the $\lambda$-line intersect 
(a)
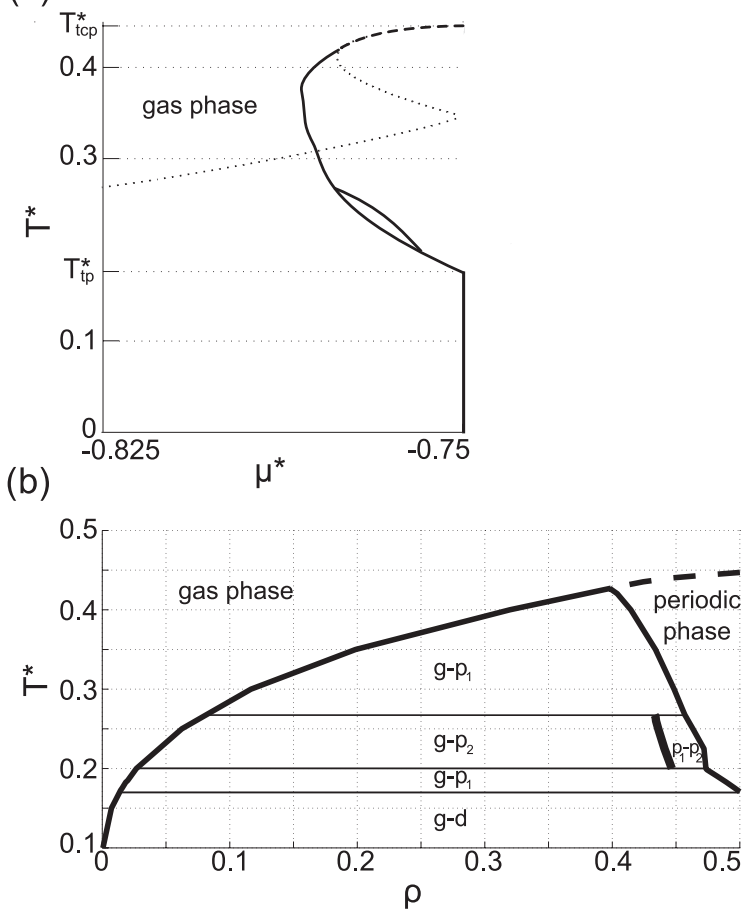

FIG. 5. MF phase diagram for $J^{*}=1 / 4$ in variables $\left(\mu^{*}, T^{*}\right)$ (a) and $\left(\rho, T^{*}\right)$ (b). The symmetry axis in (a) is $\mu^{*}=-3 / 4$ and $\rho=1 / 2$ in (b). Only half of the phase diagram is shown because of the symmetry. Dashed and solid lines represent continuous and first-order transitions. The dotted line is the $\lambda$-line. The coexisting phases in the two-phase regions in (b) are labeled by $g$ for gas, $d$ for dense fluid, and $p_{1}, p_{2}$ for the periodic phases with the smaller and the larger period, respectively. The density range of stability of the large-period phase is within the thickness of the line. The periodic phases in (a) are stable inside the lens $\left(p_{2}\right)$ and inside the loop $\left(p_{1}\right)$.

(see Figs. 3 and 7). The coexistence between the two periodic phases terminates at a critical point, where the densities, amplitudes, and periods of the two phases become the same. The $\left(\mu^{*}, T^{*}\right)$ and $\left(\rho, T^{*}\right)$ phase diagrams for $J^{*}=3$ are shown in Fig. 7. The amplitudes of the two periodic phases along their coexistence line and for $T^{*}=0.347$ are shown in Fig. 8 as functions of $T^{*}$ and $\mu^{*}$, respectively. The density profiles in the two periodic phases for selected thermodynamic states are shown in Fig. 9. As far as we know, coexistence of two ordered phases with the same symmetry but different degree of order has not been reported yet.

\section{EXACT SOLUTIONS}

\section{A. Transfer matrix and exact expressions}

Since the range of interactions is 3 , we coarse-grain the lattice and introduce $L / 3$ boxes consisting of three neighboring lattice sites, and labeled by integer $1 \leq k \leq L / 3$. The microstates in the $k$ th box are

$$
\hat{S}(k)=(\hat{\rho}(3 k-2), \hat{\rho}(3 k-1), \hat{\rho}(3 k)) .
$$

There are $2^{3}$ possible microstates in each box. We distinguish 4 states with the first site occupied and the remaining sites either occupied or empty, and denote such states by $\hat{S}_{1}(k)$ $=(1, \hat{\rho}(3 k-1), \hat{\rho}(3 k))$. Likewise, we denote states with the second site occupied by $\hat{S}_{2}(k)=(\hat{\rho}(3 k-2), 1, \hat{\rho}(3 k))$,

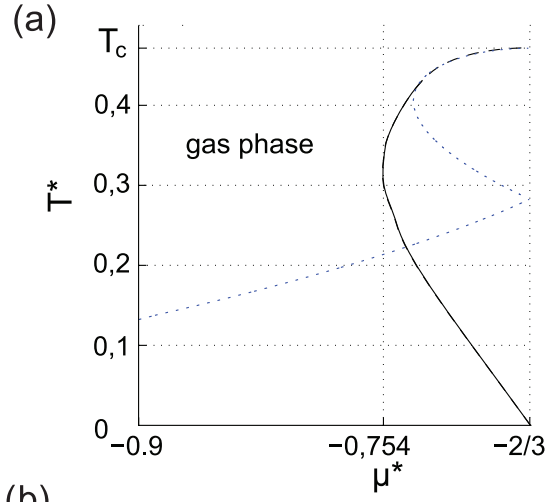

(b)

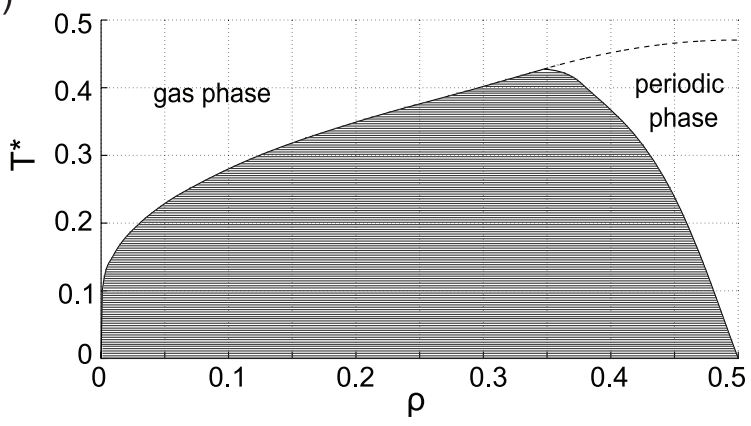

FIG. 6. MF phase diagram for $J^{*}=1 / 3$ in variables $\left(\mu^{*}, T^{*}\right)$ (a) and $\left(\rho, T^{*}\right)$ (b). The symmetry axis is $\mu^{*}=-2 / 3$ and $\rho=1 / 2$ in (a) and (b), respectively. Only half of the phase diagram is shown because of the symmetry. Dashed and solid lines represent continuous and first-order transitions between the disordered fluid and the periodic phase. The dotted line is the $\lambda$-line. The periodic phase in (a) is stable inside the loop (thick line). The two-phase regions in (b) are shaded.

and with the third site occupied by $\hat{S}_{3}(k)=(\hat{\rho}(3 k-2)$, $\hat{\rho}(3 k-1), 1)$.

The Hamiltonian of the system with PBC can be written in the form:

$$
H^{*}[\{\hat{\rho}(x)\}]=\sum_{k=1}^{L / 3} H_{t}^{*}[\hat{S}(k), \hat{S}(k+1)],
$$

where

$$
\begin{aligned}
H_{t}^{*}[\hat{S}(k), \hat{S}(k+1)]= & \sum_{x=3 k-2}^{3 k}[-\hat{\rho}(x) \hat{\rho}(x+1) \\
& \left.+J^{*} \hat{\rho}(x) \hat{\rho}(x+3)-\mu^{*} \hat{\rho}(x)\right] .
\end{aligned}
$$

We introduce a $8 \times 8$ transfer matrix $\mathbf{T}$ with the matrix elements:

$$
\begin{aligned}
T(\hat{S}(k), \hat{S}(k+1)) & \equiv e^{-\beta^{*} H_{t}^{*}[\hat{S}(k), \hat{S}(k+1)]} \\
& =\sum_{i=1}^{8} P_{i}(\hat{S}(k)) \lambda_{i} P_{i}^{-1}(\hat{S}(k+1)),
\end{aligned}
$$

where the eigenvalues of $\mathbf{T}$ are denoted by $\lambda_{i}$ such that $\left|\lambda_{i}\right|$ $\geq\left|\lambda_{i+1}\right|$, the elements $(\hat{S}, i)$ of the matrix $\mathbf{P}$ transforming $\mathbf{T}$ to its eigenbasis are denoted by $P_{i}(\hat{S})$, and the elements $(i, \hat{S})$ of the matrix inverse to $\mathbf{P}$ by $P_{i}^{-1}(\hat{S})$. Note that $\mathbf{T}$ is not symmetric, hence pairs of complex-conjugate eigenvalues may occur. However, because $\mathbf{T}$ is a finite matrix with positive elements, from the Frobenius theorem it follows that the largest (in absolute value) eigenvalue is non-degenerate. 
(a)

(b)
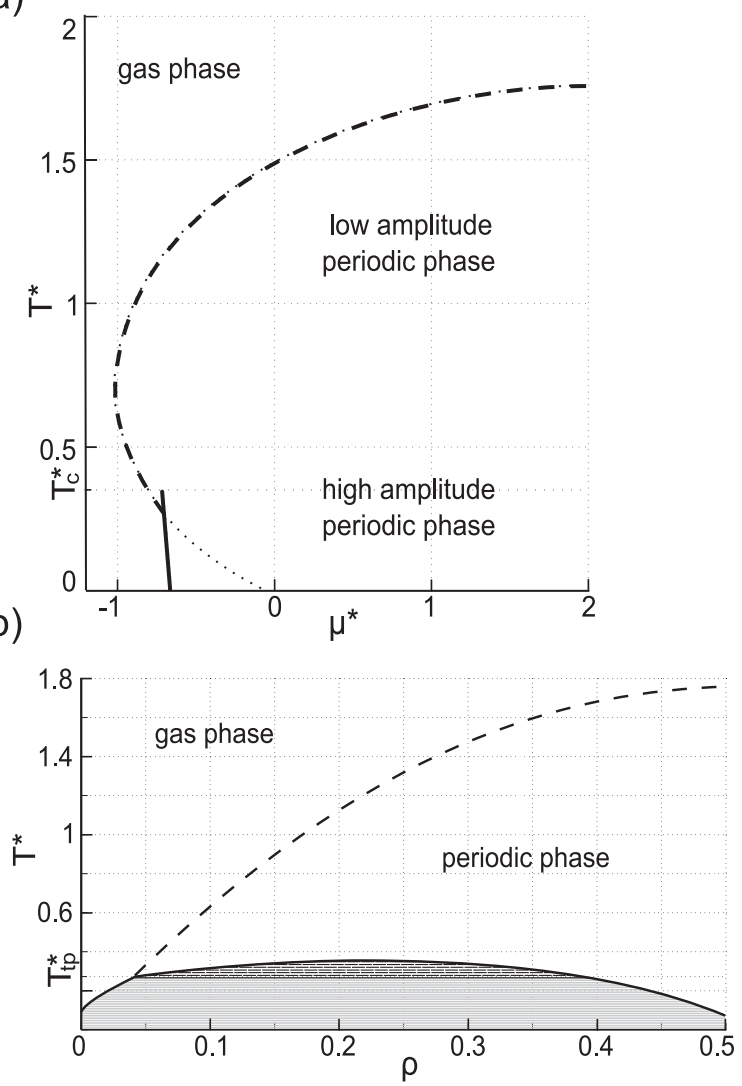

FIG. 7. MF phase diagram for $J^{*}=3$ in variables $\left(\mu^{*}, T^{*}\right)$ (a) and $\left(\rho, T^{*}\right)$ (b). The symmetry axis is $\mu^{*}=2$ and $\rho=1 / 2$ in (a) and (b), respectively. Only half of the phase diagram is shown because of the symmetry. Dashed and solid lines represent continuous and first-order transitions. The dotted line is the $\lambda$-line. The two-phase regions in (b) are shaded with different shades for different phase equilibria. The high-amplitude periodic phase coexists with gas (for $\mu^{*}<2$ or $\rho<1 / 2$ ) or liquid (for $\mu^{*}>2$ or $\rho>1 / 2$ ) for $T^{*}<T_{t p}^{*}$, and with the low-amplitude periodic phase for $T^{*}>T_{t p}^{*}$. The coexistence line between the two periodic phases (short solid line above the dashed line in (a)) begins at $T^{*}=T_{t p}^{*}$ and terminates at the critical point with $T_{c}^{*} \approx 0.34713$. Note that the point where the transition between the disordered and the periodic phases changes order is not the TCP. The disordered phase coexists with one periodic phase for $T^{*}<T_{t p}^{*}$, and undergoes a continuous transition to the other periodic phase for $T^{*}>T_{t p}^{*}$, whereas at the TCP the transition between the same phases changes order.

The grand statistical sum in terms of the transfer matrix takes the form:

$$
\Xi=\operatorname{Tr} \mathbf{T}^{L / 3}=\sum_{i=1}^{8} \lambda_{i}^{L / 3}
$$

and for the grand potential we obtain

$$
\begin{aligned}
\Omega^{*} / L & =-p^{*}=-T^{*}\left[\frac{1}{3} \ln \lambda_{1}+\frac{1}{L} \ln \left(1+\sum_{i=2}^{8}\left(\frac{\lambda_{i}}{\lambda_{1}}\right)^{L / 3}\right)\right] \\
& \simeq{ }_{L \rightarrow \infty}-\frac{T^{*}}{3} \ln \lambda_{1} .
\end{aligned}
$$

In the case of PBC the average density is independent of the position, $\bar{\rho}=\langle\hat{\rho}(1)\rangle$. From the definition of the average (a)

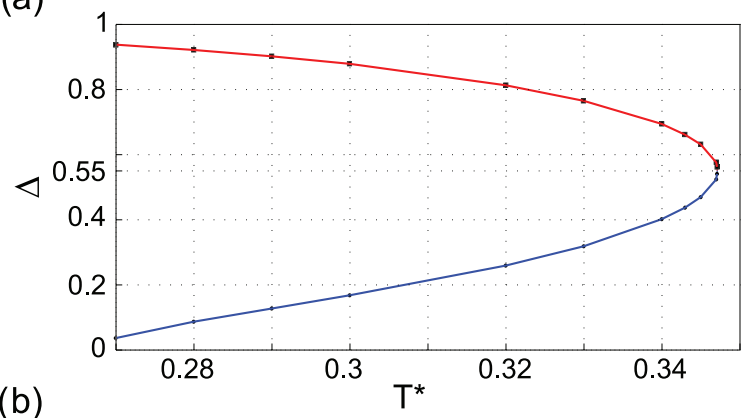

(b)

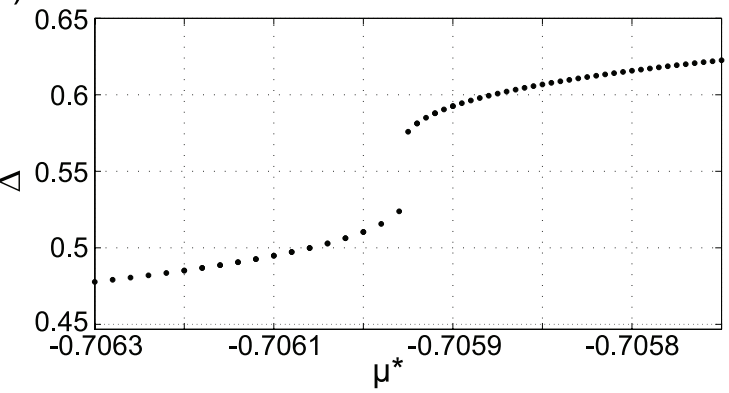

FIG. 8. Amplitudes of the density profiles in the two periodic phases for $J^{*}$ $=3$. (a) As a function of temperature along the coexistence line (the lines meet at $T_{c}^{*} \approx 0.34713$ ); (b) as a function of $\mu^{*}$ for $T_{c}^{*}=0.347$.
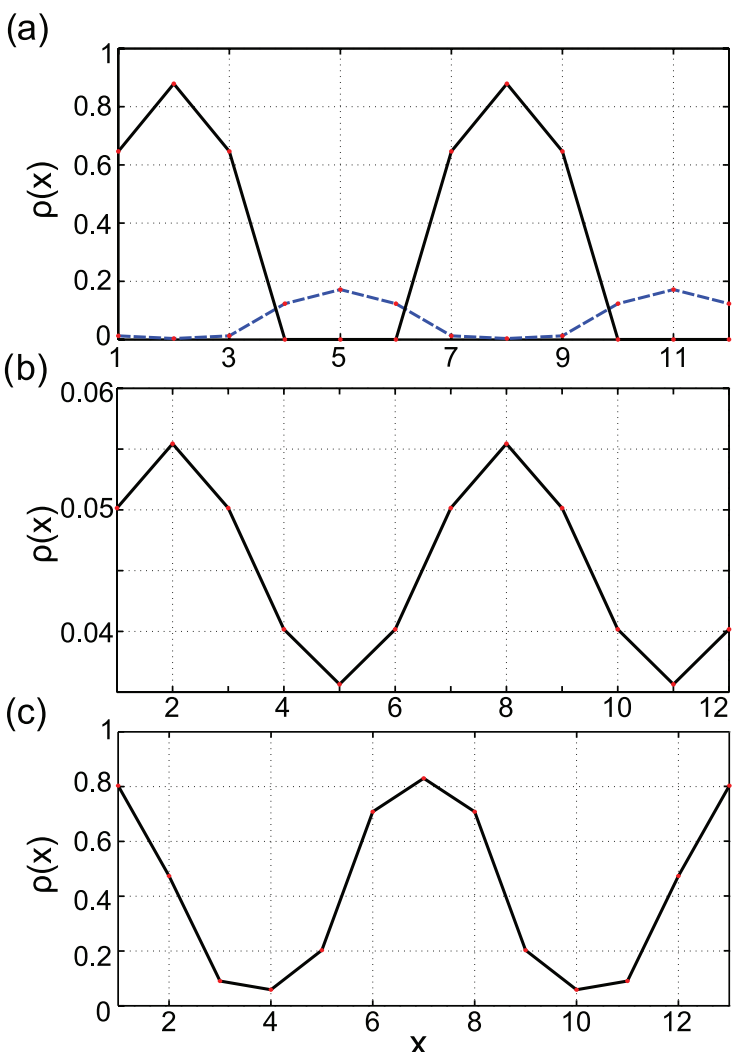

FIG. 9. Density profiles (a) in the coexisting high- and low-amplitude phases for $T^{*}=0.3$. The lines are shifted horizontally for clarity. (b) In the lowamplitude phase close to the continuous transition to the fluid at $T^{*}=0.3$ and (c) for $T^{*}=1.3$ and $\mu^{*}=1$. The quasi-periodic structure with a period incommensurate with the lattice is obtained from a density profile with a large-period when $2 \pi / k_{b}$ is noninteger. The lines connecting the results for integer $x$ are to guide the eyes. 
density and from Eqs. (23) and (24) we obtain

$$
\begin{aligned}
\langle\hat{\rho}(1)\rangle & =\frac{\sum_{\hat{S}_{1}(1)} \sum_{i=1}^{8} \lambda_{i}^{L / 3} P_{i}^{-1}\left(\hat{S}_{1}(1)\right) P_{i}\left(\hat{S}_{1}(1)\right)}{\sum_{i=1}^{8} \lambda_{i}^{L / 3}} \\
& \simeq L \rightarrow \infty \sum_{\hat{S}_{1}(1)} P_{1}^{-1}\left(\hat{S}_{1}(1)\right) P_{1}\left(\hat{S}_{1}(1)\right)
\end{aligned}
$$

Let us consider the correlation function for the sites separated by a distance $x=3 k+i$, where $k \geq 0$ and $i=0,1$, 2 . Because the interaction range is 3 , and the transfer matrix operates between triples of sites, we shall obtain different expression for $\langle\hat{\rho}(1) \hat{\rho}(1+x)\rangle$ for different $i=0,1,2$. We introduce the notation:

$$
G(3 k+i)=\langle\hat{\rho}(1) \hat{\rho}(1+3 k+i)\rangle-\langle\hat{\rho}(1)\rangle^{2} .
$$

From the definition of $\left\langle\hat{\rho}(x) \hat{\rho}\left(x^{\prime}\right)\right\rangle$ and from Eqs. (23) and (26) we obtain the asymptotic expression for $L \rightarrow \infty$ :

$$
G(3 k+i)=\sum_{n=2}^{8}\left(\frac{\lambda_{n}}{\lambda_{1}}\right)^{k} A_{1}^{(n)} B_{1+i}^{(n)},
$$

where $i=0,1,2$,

$$
\begin{aligned}
& A_{j}^{(n)}=\sum_{\hat{S}_{j}} P_{n}\left(\hat{S}_{j}\right) P_{1}^{-1}\left(\hat{S}_{j}\right), \\
& B_{j}^{(n)}=\sum_{\hat{S}_{j}} P_{n}^{-1}\left(\hat{S}_{j}\right) P_{1}\left(\hat{S}_{j}\right),
\end{aligned}
$$

and $\hat{S}_{j}$ is defined below Eq. (20). The asymptotic decay of correlations for $k \gg 1$ is determined by the eigenvalue $\lambda_{2}$ with the second largest absolute value.

If $\lambda_{2}$ is real, then for $k \gg 1$ and $i=0,1,2$ we can write

$$
G(3 k+i)=\left(\operatorname{sgn}\left(\lambda_{2}\right)\right)^{k} e^{-3 k / \xi} A_{1}^{(2)} B_{1+i}^{(2)},
$$

where the correlation length is

$$
\xi=3 / \ln \left(\frac{\lambda_{1}}{\left|\lambda_{2}\right|}\right) .
$$

Note the qualitatively different behavior for $\lambda_{2}>0$ and $\lambda_{2}$ $<0$. For $\lambda_{2}<0$ the correlation function changes sign when the separation between the particles increases by 3 , in analogy with the density of the periodic phase in the ground state. The case $\lambda_{2}>0$ corresponds to decay of correlations in the gas or liquid phases where no clusters consisting of three particles separated by 3 vacancies are formed.

If $\lambda_{2}$ is complex, then $\lambda_{3}=\lambda_{2}^{*}, A_{1}^{(3)}=A_{1}^{(2) *}$, and $B_{j}^{(3)}$ $=B_{j}^{(2) *}$. We introduce the notation:

$$
\lambda_{2}=\left|\lambda_{2}\right| e^{i \lambda}, \quad A_{1}^{(2)}=\left|A_{1}^{(2)}\right| e^{i \alpha_{1}}, \quad B_{j}^{(2)}=\left|B_{j}^{(2)}\right| e^{i \beta_{j}},
$$

and for $k \gg 1$ and $i=0,1,2$ obtain the asymptotic expression:

$$
G(3 k+i)=\mathcal{A}_{i} e^{-3 k / \xi} \cos \left(k \lambda+\theta_{i}\right),
$$

where $\mathcal{A}_{i}=2\left|A_{1}^{(2)}\right|\left|B_{1+i}^{(2)}\right|$ and $\theta_{i}=\alpha_{1}+\beta_{1+i}, i=0,1,2$. Similar expression was proposed in Ref. 41 for a 1D and in Ref. 18 for a 3D system. The structure factor obtained in experiments and theory ${ }^{3,8,13-23}$ is also consistent with this form.
In general, $-\pi \leq \lambda \leq \pi$, and $2 \pi / \lambda$ is noninteger. Except for $\lambda$ $= \pm \pi$ (but in this case the imaginary part of $\lambda_{2}$ vanishes), the period of the exponentially damped oscillations is incommensurate with the lattice. This is similar to the results of the MF stability analysis and to the incommensurate density profiles obtained in MF for higher temperatures.

When $\lambda= \pm \pi \mp \epsilon$ with $\epsilon \ll 1$, then we can write Eq. (34) in the equivalent form:

$$
G(3 k+i)=(-1)^{k} e^{-3 k / \xi} \mathcal{G}(k, i)
$$

with

$$
\mathcal{G}(k, i)=\mathcal{A}_{i} \cos \left(6 \pi k / w+\phi_{i}\right),
$$

where the phase and the period of the amplitude modulations are $\phi_{i}=-\theta_{i} \operatorname{sgn}(\lambda)$ and

$$
w=\frac{6 \pi}{|\lambda-\operatorname{sgn}(\lambda) \pi|} .
$$

The first factor in (35) changes sign when the distance increases by 3 . The last factor describes the modulated amplitude with the wavelength of modulations $w \gg 6$ if $\lambda \rightarrow \pm \pi$.

We have obtained $\lambda_{i}$ and the matrix $\mathbf{P}$ numerically for different $J^{*}, \mu^{*}$, and $T^{*}$ and the results are presented in Sec. IV B.

\section{B. Results}

There are no phase transitions in a thermodynamic sense in one-dimensional systems. However, instead of a discontinuity, a rapid change of the density as a function of $\mu^{*}$ or $p^{*}$ can occur. Moreover, instead of long-range order and the associated periodic density, a short-range order with exponentially damped oscillatory decay of correlations with very large correlation length may exist. In order to verify if such pseudo phase transitions occur in this model, we calculate density and pressure for several values of $J^{*}$ for the range of $\mu^{*}$ and $T^{*}$ corresponding to the phase transitions obtained in MF. In the next step we examine the correlation functions.

\section{Thermodynamic properties (equation of state)}

In Figs. 10 and $11 p\left(\mu^{*}\right)$ and $\rho\left(\mu^{*}\right)$ obtained from Eqs. (25) and (26) are shown for $J^{*}=3$ and $J^{*}=1 / 4$ for 0.05 $<T^{*}<1$. By eliminating $\mu^{*}$ from Eqs. (25) and (26) we obtain the EOS, and present several isotherms $\rho\left(p^{*}\right)$ in Figs. 12 and 13 . The chosen strengths of the repulsion to attraction ratio correspond to qualitatively different ground state and MF phase diagrams (see Figs. 1, 7, and 5). Let us first discuss $J^{*}$ $=3$. For low $T^{*}$ one can observe that although $p^{*}\left(\mu^{*}\right)$ is a smooth function, its slope changes rapidly for the two values of $\mu^{*}$ that correspond to the phase transitions at $T^{*}=0$ and in MF. In accordance with this behavior the density changes from nearly 0 to $1 / 2$ in a very narrow range of $p^{*}$ and $\mu^{*}$, remains nearly constant for large intervals of $p^{*}$ and $\mu^{*}$, and again changes rapidly from $\rho=1 / 2$ to $\rho \approx 1$. Very large compressibility for $\rho \neq 1 / 2$ changes to very small compressibility for $\rho \approx 1 / 2$. It is necessary to substantially increase the pressure in order to induce a slight increase of the density from $\rho=1 / 2$, and a slight further increase of pressure is sufficient 


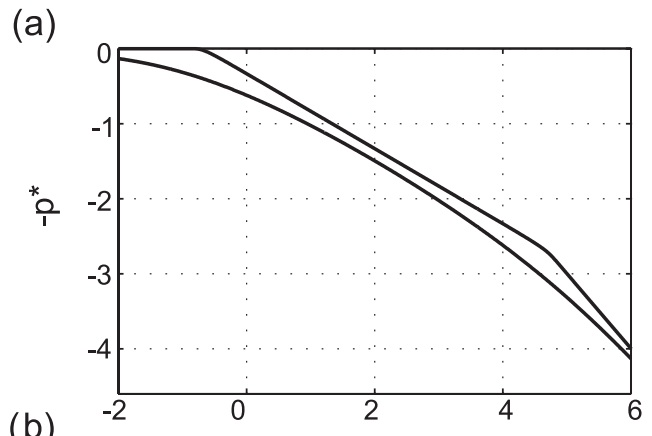

(b)

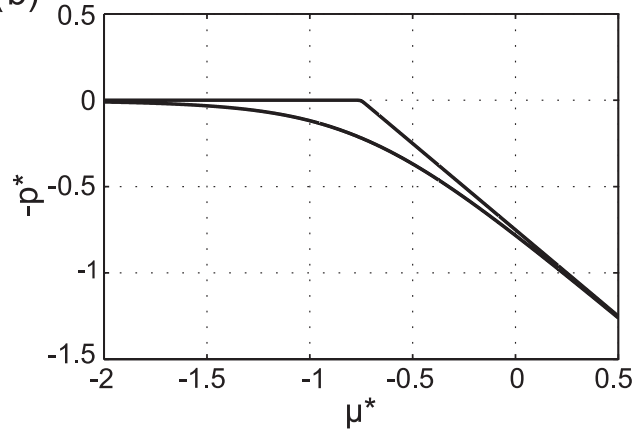

FIG. 10. $p\left(\mu^{*}\right)$ obtained from Eq. (25) (a) $J^{*}=3$; top line: $T^{*}=0.1$, bottom line: $T^{*}=1$ and (b) $J^{*}=1 / 4$; top line: $T^{*}=0.05$, bottom line: $T^{*}=0.5$.

for a rapid compression to $\rho \approx 1$. When $T^{*}$ increases from $T^{*}$ $\approx 0.1$, the density changes from the gas density to $1 / 2$ more and more gradually. For $T^{*}>0.5$ there are no abrupt changes of the slopes of the $\rho\left(p^{*}\right)$ and $\rho\left(\mu^{*}\right)$ lines, but the curvature of these lines is significantly smaller than in the one-phase region of a simple fluid. This is because the repulsion between the particles at the distance 3 leads to a significant increase of pressure for random distribution of particles. On the other hand, small pressure for $\rho<1 / 2$ when $T^{*}$ is low signals that in majority of states clusters made of at most 3 particles are separated by at least 3 empty sites. Similar behavior is observed for $1<J^{*}<3$ (Fig. 13), but the range of $\mu^{*}, p^{*}$, and $T^{*}$ for which $\rho \approx 1 / 2$ and remains nearly constant decreases with decreasing $J^{*}$. For $J^{*}<1$ the plateau at the $\rho\left(\mu^{*}\right)$ and $\rho\left(p^{*}\right)$ curves for $\rho=1 / 2$ disappears.
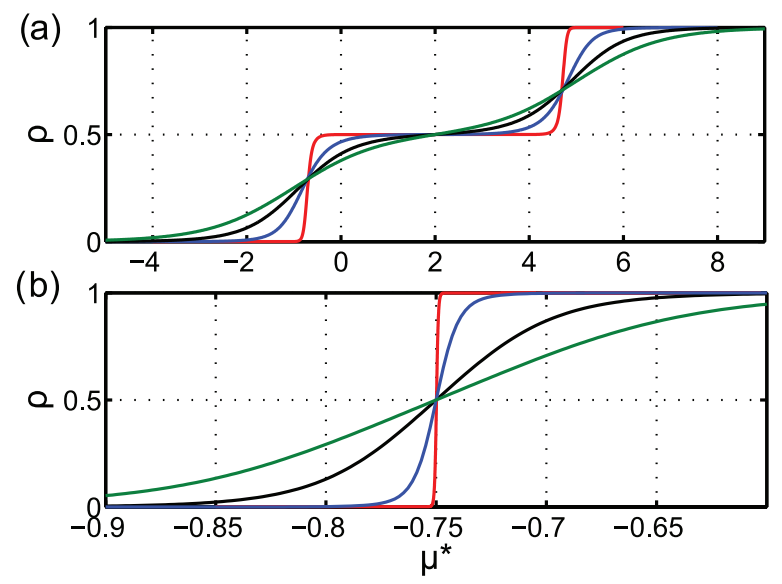

FIG. 11. $\rho\left(\mu^{*}\right)$ obtained from Eq. (26) for $J^{*}=3$ and $T^{*}=0.1,0.4,0.7,1$ (top to bottom line on the right) (a) and $J^{*}=1 / 4$ and $T^{*}=0.005,0.05,0.1$, 0.15 (top to bottom line on the right) (b).

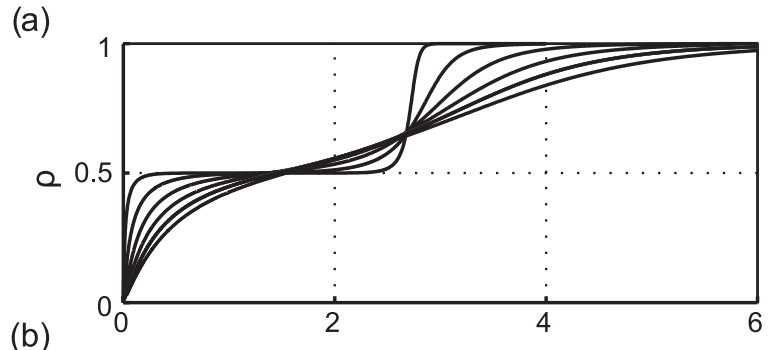

(b)

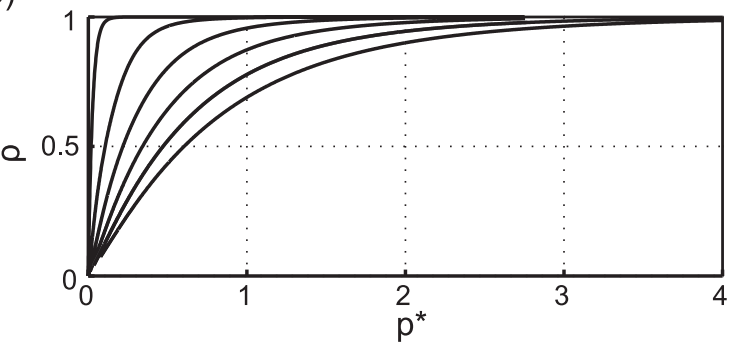

FIG. 12. $\operatorname{EOS} \rho\left(p^{*}\right)$ isotherms obtained from Eqs. (25) and (26) for $T^{*}=$ $0.1,0.2,0.3,0.4,0.5$, and 1 (top to bottom line on the left) for $J^{*}=3$ (a) and $J^{*}=1 / 4(\mathrm{~b})$.

For $J^{*}=1 / 4$ we can see in Fig. 10(b) a rapid change of the slope of the $p^{*}\left(\mu^{*}\right)$ line and in Fig. 11 the corresponding change of density from $\rho \approx 0$ to $\rho \approx 1$ when $T^{*}<0.06$. For $T^{*}>0.15$ the shapes of $p^{*}\left(\mu^{*}\right), \rho\left(\mu^{*}\right)$, and $\rho\left(p^{*}\right)$ (Fig. 12) resemble the corresponding curves in the single-phase simple fluid. We thus see a pseudo-transition between the gas and liquid phases for very low $T^{*}$. We conclude that the thermodynamic properties show no signature of the weakly ordered periodic phase previously found in MF (Fig. 5). By comparing Figs. 12(a) and 12(b) one can see the much lower pressure in this case than for the repulsion-dominated case of $J^{*}=3$.

\section{Structure (correlation function)}

Our aim in this section is to discuss the exact results for the correlation function for $J^{*}=3$ and $J^{*}=1 / 4$, corresponding to qualitatively different ground state (Fig. 1) and MF phase diagrams (Figs. 7 and 5). We particularly address the question for what parameters the periodic order occurs, and

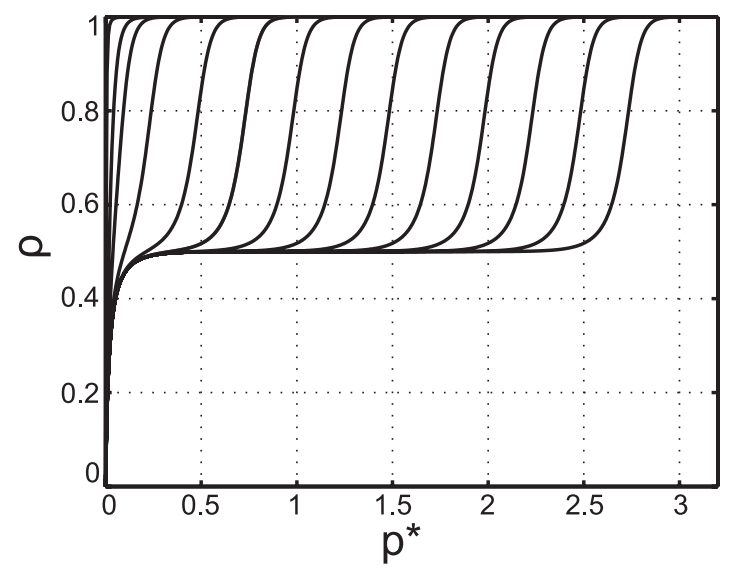

FIG. 13. EOS $\rho\left(p^{*}\right)$ isotherms obtained from Eqs. (25) and (26) for $T^{*}=$ 0.1 . From the left to the right line $J^{*}=0.1,0.25,1 / 3,0.5,0.75, \ldots, 2.75,3$. 


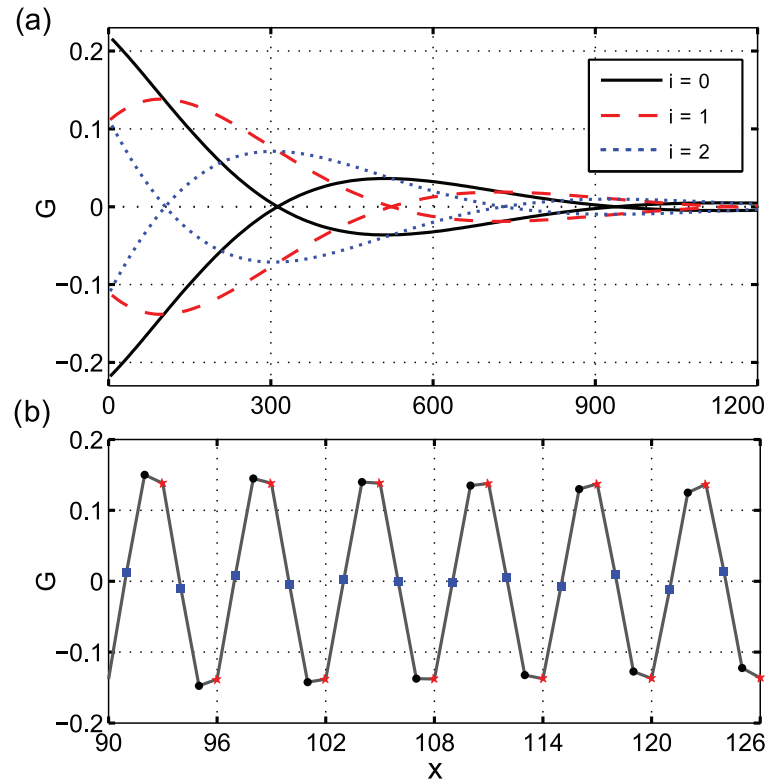

FIG. 14. The correlation function $G(x)$ for $x=3 k+i$ with $i=0,1,2$ (Eq. (34)) for $J^{*}=3, \mu^{*}=0$, and $T^{*}=0.1$ (inside the MF stability region of the periodic phase). Solid line and the circles (black), dashed line and the asterisks (red), and dotted line and the squares (blue) correspond to $i=$ $0,1,2$, respectively. The bottom panel shows a small portion of the upper panel.

how the range and amplitude of the correlation function depends on $\mu^{*}, T^{*}$, and $J^{*}$.

For $J^{*}=3$ we obtain complex $\lambda_{2}$ for the considered region of $\left(\mu^{*}, T^{*}\right)$. In this case the correlation function is given in Eq. (34), and presented in Figs. 14 and 15. In Fig. $14 \mu^{*}$ corresponds to $\rho \approx 1 / 2$, where the periodic phase is predicted in MF, and in Fig. $15 \mu^{*}$ corresponds to $\rho \approx 0$ (homogeneous gas in MF).

The correlation length $\xi$ (Eq. (32)) and the amplitude of the correlation function $\mathcal{A}_{0}$ (see below Eq. (34)) are shown in Figs. 16 and 17, respectively. For $\mu \leq-2 / 3$ the wavenumber $\lambda$ is shown in Fig. 18(a), and for $\mu \geq-2 / 3$ the period $w$ of modulations of the amplitude (see (35) and (36)) is shown in Fig. 18(b).

From Fig. 16(a) it follows that for $\mu^{*}<-2 / 3$ the correlation length $\xi$ first increases slightly for decreasing $T^{*}$, but

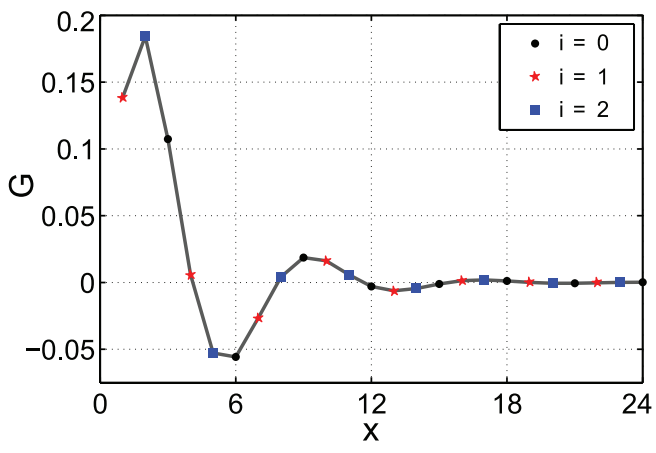

FIG. 15. The correlation function $G(x)$ for $x=3 k+i$ with $i=0,1,2$ (Eq. (34)) for $J^{*}=3, \mu^{*}=-0.7$, and $T^{*}=0.1$ (outside the MF stability region of the periodic phase). Black (circle), red (asterisk), and blue (square) symbols correspond to $i=0,1,2$, respectively.

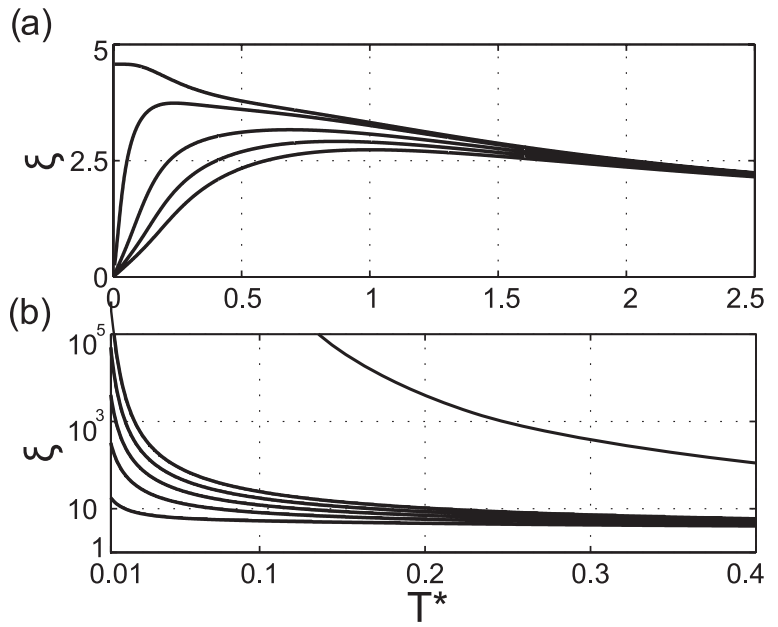

FIG. 16. The correlation length $\xi$ (Eq. (32)) for $J^{*}=3$ as a function of $T^{*}$ (a) outside the MF stability region of the periodic phase. From the top to the bottom line $\mu^{*}=-2 / 3,-0.7,-0.8,-0.9,-1$, and (b) inside the MF stability region of the periodic phase. From the bottom to the top line $\mu^{*}=-0.65,-0.6,-0.55,-0.5,-0.45$, and 2 .
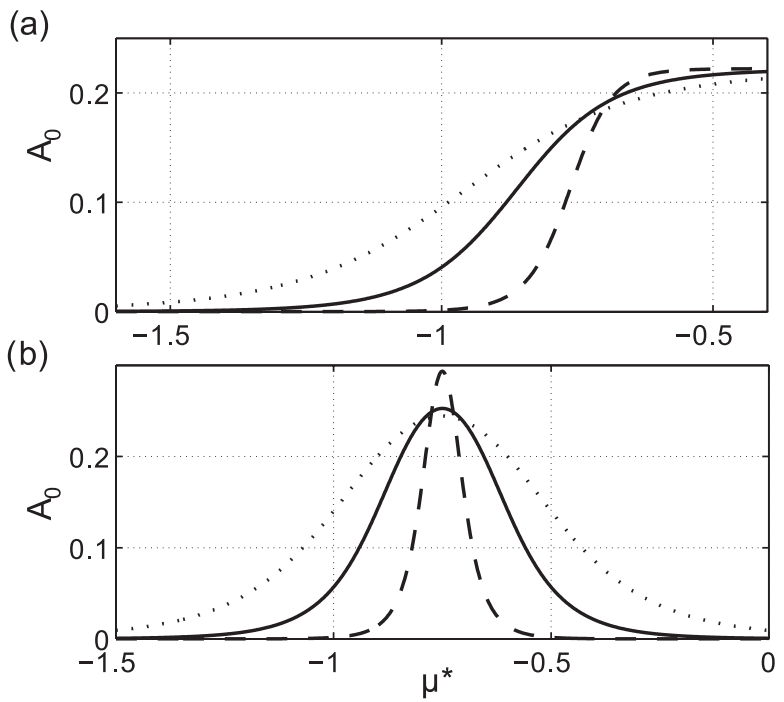

FIG. 17. The amplitude $\mathcal{A}_{0}$ of the correlation function (see Eq. (34) and below) as a function of $\mu^{*}$. Dashed, solid, and dotted lines correspond to $T^{*}$ $=0.1,0.2,0.3$, respectively. $J^{*}=3$ (a) and $J^{*}=1 / 4$ (b).
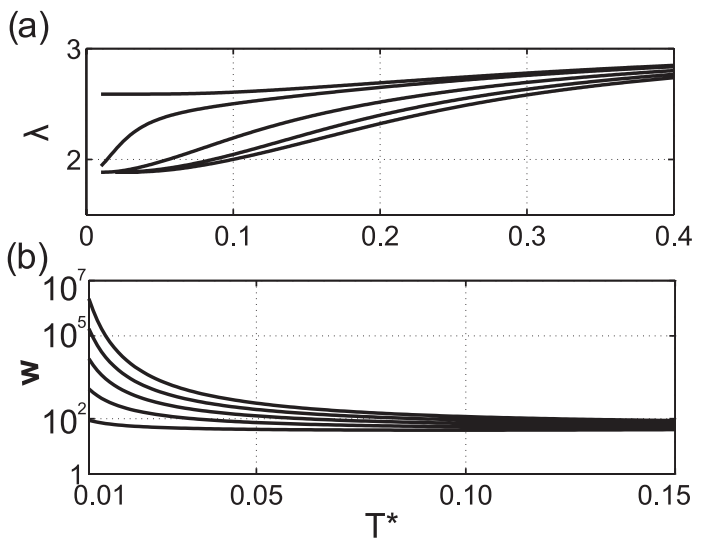

FIG. 18. (a) The wavenumber $\lambda$ of the correlation function (Eq. (34)). $J^{*}=3$ and $\mu^{*}=-2 / 3,-0.7,-0.8,-0.9,-1$ from the top to the bottom line (b) the period $w$ (Eqs. (35)-(37)) of the amplitude modulations. $J^{*}=3$ and $\mu^{*}=-0.65,-0.6,-0.55,-0.5,-0.45$ from the bottom to the top line. 
starting from $T^{*}$ depending on $\mu^{*}$ decreases rapidly to 0 for $T^{*}$ decreasing to 0 . Analogous behavior is predicted for $\mu^{*}>$ $14 / 3$ by the model symmetry. For $-2 / 3<\mu^{*}<14 / 3$ (stability of the periodic phase for $T^{*}=0$ ) the correlation length increases for decreasing $T^{*}$. For given $T^{*}$ the correlation length increases with increasing $\mu^{*}$ when $\mu^{*}<2$ and assumes a maximum for $\mu^{*}=2$. The maximum of $\xi$ is very large for $T^{*}$ $<0.15$. For $\xi \sim 10^{5}$ the range of the "short-range order" is in fact macroscopic. For particles with a diameter $10 \mathrm{~nm}$ the periodic arrangement persists to distances $\sim 1 \mathrm{~mm}$. The amplitude of the correlation function for $T^{*} \leq 0.1$ increases sharply from a very small value for $\mu^{*}<-1$ to $\sim 0.2$ for $\mu^{*}>-0.7$ (see Fig. 17(a)). The period $w$ of the modulations of the correlation function (Eqs. (35)-(37)) increases for decreasing $T^{*}$, indicating more and more ordered structure (see Fig. 18(b)). All these results confirm a qualitative change of the structure along the lines $\mu^{*}=-2 / 3,14 / 3$ for low $T^{*}$. For $-2 / 3<\mu^{*}$ $<14 / 3$ quasi long-range order with the very large correlation length and the amplitude that for low $T^{*}$ rapidly decreases at the boundaries of this region exists. From Figs. 16 and 17 we can see that the increasing correlation length and amplitude for increasing $T^{*}$ when $-1<\mu^{*}<-2 / 3$ indicates a change from a less to a more ordered structure when $T^{*}$ increases. The rapid increase of the amplitude as a function of $\mu^{*}$ for $\mu^{*} \approx-2 / 3$ near $T^{*}$ corresponding to the maximum of $\xi$ resembles the transition between the periodic phases with weak and strong order found in MF (compare Figs. 17(a) and $8(\mathrm{~b}))$. When $T^{*}$ further increases, the properties of the correlation function change more gradually and the correlation length becomes short, in consistency with the continuous transition between the ordered and disordered phases found in MF for high $T^{*}$. Despite the absence of the phase transition in the strict sense, we can see a change from a quasi-ordered periodic structure to the structure with much lower degree of order.

For $J^{*}=1 / 4$ we obtain $\lambda_{2}$ that is a real number on the low- $T^{*}$ side of a line $T_{\text {cross }}^{*}\left(\mu^{*}\right)$, and a complex number for $T^{*}$ above this line. As a result, a monotonic decay of correlations is found for $T^{*}<T_{\text {cross }}^{*}\left(\mu^{*}\right)$, and an oscillatory decay sets in for $T^{*}>T_{\text {cross }}^{*}\left(\mu^{*}\right)$. The derivatives of the correlation length $\xi$ and the wavenumber $\lambda$ with respect to $T^{*}$ have a discontinuity when the imaginary part of $\lambda_{2}$ appears (see Fig. 19). The amplitude of the correlation function (Fig. 17(b)) increases from a very small value for $\mu^{*}<-1$ to $\sim 0.3$ for $\mu^{*}=-0.75$ for $T^{*}=0.1$. For larger $T^{*}$ the increase of the amplitude is more gradual. The correlation function shown in Fig. 20 confirms that for low $T^{*}$ the correlations decay monotonically, whereas for higher $T^{*}$ the oscillatory decay of correlations is present. The correlation length, however, is rather short, as shown in Fig. 19. The monotonic decay of correlations for low $T^{*}$, and oscillatory decay of correlations at higher $T^{*}$ together with a rather rapid increase of the amplitude of the correlation function from a very small to a rather large value for some range of $\mu^{*}$ around $\mu^{*}=-3 / 4$ bear some similarity to the MF phase diagram. However, due to the much shorter correlation length than for $J^{*}=3$, we can conclude that the weak periodic order (small amplitude of density oscillations) found in MF for relatively large $T^{*}$ does not resemble an ordered phase in 1D.
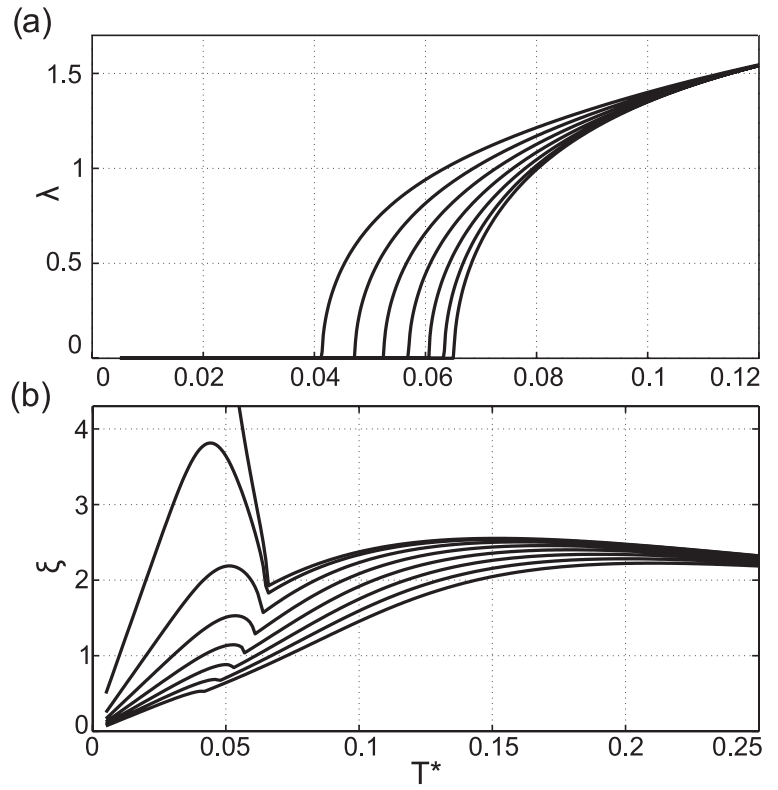

FIG. 19. The wavenumber $\lambda$ (a) and the correlation length $\xi$ (Eq. (32)) (b) of the correlation function (Eq. (34)) as a function of $T^{*}$ for $J^{*}=1 / 4$. From the bottom to the top line in (a) and from the top to the bottom line in (b) $\mu^{*}$ $=-0.75,-0.75 \pm 0.01,-0.75 \pm 0.02, \ldots,-0.75 \pm 0.07$.

\section{MC SIMULATIONS IN CANONICAL ENSEMBLE}

In Sec. IV we have shown that the disordered phase in systems with competing interactions is characterized by the EOS and the correlation function that for strong repulsion are completely different than in simple fluids. In this section we would like to determine how the pretransitional ordering into clusters whose positions can be correlated over very large distances is reflected in the measurable thermal properties such as the constant-volume specific heat. Since analytical calculations in canonical ensemble are very difficult, we perform MC simulations.
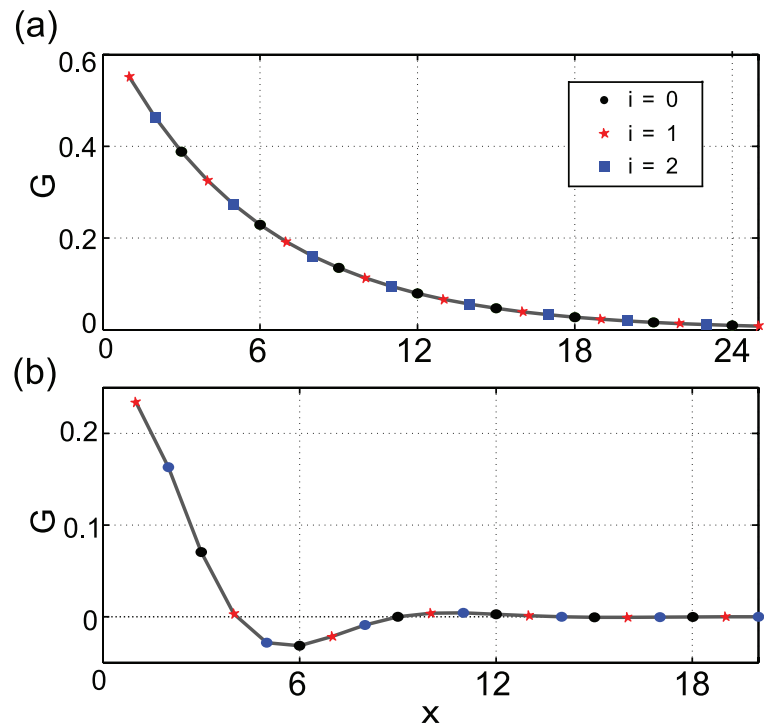

FIG. 20. The correlation function $G(x)$ for $x=3 k+i$ with $i=0,1,2$ (Eq. (34)) for $J^{*}=1 / 4$ and $\mu^{*}=-3 / 4$ for $T^{*}=0.05$ (a) and $T^{*}=0.2$ (b). Black (circle), red (asterisk), and blue (square) symbols correspond to $i$ $=0,1,2$, respectively. 


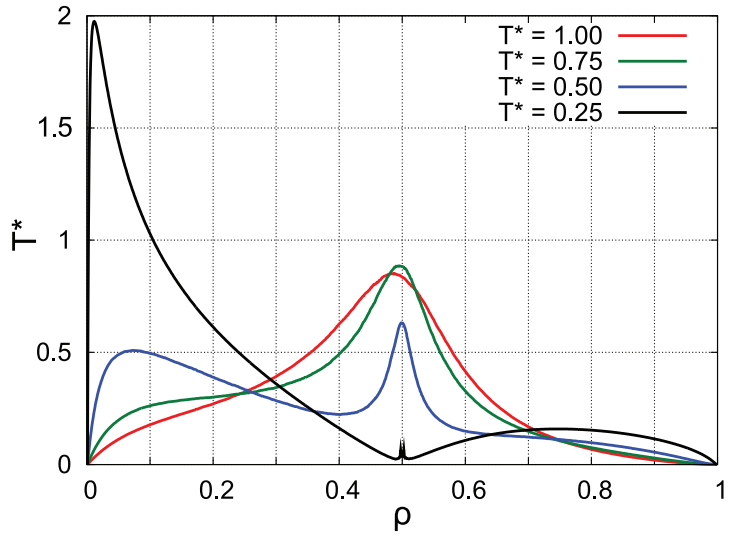

FIG. 21. The specific heat per particle (in $k_{B}$ units) as a function of density (dimensionless) for $J^{*}=3$. From the top to the bottom line on the left $T^{*}=0.25,0.5,0.75,1$ with $L=1200$.

The basic step in the sampling is made as follows: Given the current configuration of the system one chooses at random with equal probability one of the occupied positions, $x$ (with $\hat{\rho}(x)=1$ ), and one of the empty positions $x^{\prime}$ (with $\hat{\rho}\left(x^{\prime}\right)=0$ ), the trial configuration is then constructed by swapping the states between the positions $x$ and $x^{\prime}$. Considering the energies of the current and the trial configurations one applies the Metropolis criterion ${ }^{49}$ to decide whether the trial configuration is accepted as the new configuration of the system or not. The heat capacity per particle, $c_{v}=(\partial(H / N) / \partial T)_{N, L}$, is computed using the fluctuation formula:

$$
c_{v}=\frac{1}{N k_{B} T^{2}}\left[\left\langle H^{2}\right\rangle-\langle H\rangle^{2}\right],
$$

where the angular brackets indicate averages on the canonical ensemble.

For $J^{*}=3$ the specific heat is shown in Fig. 21. The results for $J^{*}=0.25$ are shown in Fig. 22. For both values of $J^{*}$ we can observe the presence of a peak at low density. On cooling the system the height of this peak increases, and the density where it appears is shifted to lower values. This maximum in the heat capacity at low temperature can be explained as an effect of the equilibrium between isolated particles and clusters of several particles. Given the Hamiltonian

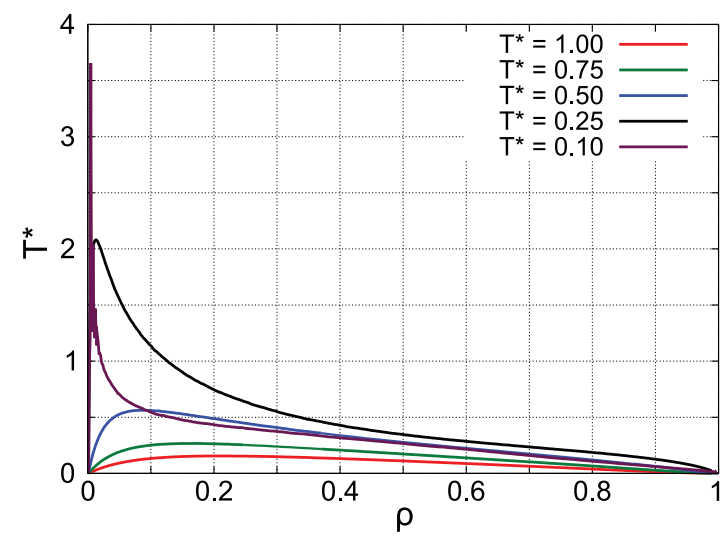

FIG. 22. The specific heat (in $k_{B}$ units) as a function of density (dimensionless) for $J^{*}=1 / 4$. From the top to the bottom line on the left $T^{*}=0.1,0.25$, $0.5,0.75,1$ with $L=840$. of the model, these clusters are likely to be triples in the case of $J^{*}=3$. At low temperature and low density the loss of entropy due to the formation of clusters is compensated by the energetic effect due to the attractive interaction between the nearest neighbors.

There are, however, significant differences between the heat capacities curves for $J^{*}=0.25$ and $J^{*}=3.0$, especially for $\rho \simeq 1 / 2$. These differences are consistent with the results for the EOS and the correlation function that indicate periodic ordering of clusters only for strong enough repulsion. For $J^{*}$ $=3.0$, at low temperature, we can observe a basin around $\rho$ $=1 / 2$, and a narrow peak centered also at $\rho=1 / 2$. Focusing in the region $\rho \leq 1 / 2$, the ground state configurations are formed by triples of occupied positions. Each triple of occupied cells is separated at least by three empty positions from another triple. Since the triple-triple interaction is repulsive at short distances, the system does not show any trend to exhibit a pseudo phase separation to form large regions of occupied and empty positions, and therefore the small energy fluctuations lead to small values of the heat capacity. Notice, however that for $J^{*}<1 / 3$ and low temperature the dominant attractive interactions lead to a condensation of particles in large clusters of occupied cells. In this case neither the basin at low $T^{*}$ nor the peak near $\rho=1 / 2$ is present.

The peak of $c_{v}(\rho)$ at $\rho \simeq 1 / 2$ for $J^{*}=3$ can be interpreted as a signature of a pseudo-phase transition between an ordered (or quasi-ordered) phase (periodic phase with $l=6$ for $\rho=1 / 2$ ) and a high temperature disordered phase. Notice that as one approaches $\rho=1 / 2$ the degeneracy of the ground state reduces sharply, then we can describe this peak as produced by the competition between the ground state (with very low entropy values when $\rho \rightarrow 1 / 2$ ) and disordered states (with higher values of energy and entropy). This ordered pseudophase lies between the fluid of small droplets $(\rho<1 / 2, T$ $\rightarrow 0)$ and the fluid of bubbles $(\rho>1 / 2, T \rightarrow 0)$. In spite of the lack of real phase transitions for one-dimensional models with short range interactions, the periodic pseudo phase with density $\rho=1 / 2$ resembles to some extent the low-density crystalline phases that appear in core-softened models. ${ }^{50}$

We conclude that measurements of $c_{v}$ in systems with competing interactions can give information on the formation and properties of clusters for very small densities, and on formation of phases (or pseudo-phases) with periodically ordered clusters for higher densities. While in the latter case thermal properties are consistent with the EOS and the correlation function, for very small densities they provide additional information on the equilibrium between isolated particles and clusters.

\section{SUMMARY AND DISCUSSION}

We have developed a generic model for self-assembly in systems with competing interactions. The $1 \mathrm{D}$ version of the model was solved in MF approximation and exactly in the grand canonical ensemble for the whole range of the repulsion to attraction ratio $J^{*}$. In addition, MC simulations have been performed in the canonical ensemble. We have found interesting and counterintuitive results even in 1D case. 
The ground state $\left(T^{*}=0\right)$ shows a sequence of phases fluid (gas)-periodic-fluid (liquid) for increasing $\mu^{*}$ when $J^{*}$ $>1 / 3$. This behavior agrees with the reentrant melting observed experimentally in several systems ${ }^{46,47}$ For weaker repulsions only the two fluid phases are present for $T^{*}=0$. A peculiar property of the MF solutions is the existence of the periodic phase for a range of $\mu^{*}$ that is broader for intermediate temperatures than at $T^{*}=0$. In the case of $1 / 9<J^{*}$ $<1 / 3$ the periodic phase appears for some range of $T^{*}$, even though it is absent for $T^{*}=0$. Usually, the increase of $T^{*}$ leads to less ordered structures, and the MF result is counterintuitive. In particular, the MF phase diagrams show that for decreasing temperatures at constant $\mu^{*}$ a sequence of phases fluid-periodic-fluid appears, i.e., we find reentrant melting.

There are no phase transitions in 1D systems. In order to find if the MF phase behavior is associated with a qualitative change of mechanical, structural, and thermal properties, we have analyzed the exact results for the EOS and correlation function and performed MC simulations for $c_{v}$. We emphasize that the information obtained via exact calculations and MC simulations on mechanical, structural, and thermal properties is consistent and complementary.

We have found that for $J^{*}>1$ the correlation function exhibits oscillatory decay with the correlation length that is a few orders of magnitude larger than the size of the particles for some region of the $\left(\mu^{*}, T^{*}\right)$ phase diagram (see Fig. 16). This indicates formation of clusters separated by distances larger than the range of the repulsion. As a result of the above ordering, the shapes of the EOS isotherms are completely different than in simple fluids (see Fig. 12). The compressibility is very large for the range of densities where the pseudo-phase transitions between the fluid and the periodic phase are expected. On the other hand, the compressibility is extremely small for $\rho \approx 1 / 2$, where quasi-periodically distributed clusters that repel each other at distances shorter than $3 \sigma$ are formed. Similar properties of the EOS can be expected for higher dimensions. The pseudo phase transition to the periodically distributed clusters is associated with a peak in the specific heat at $\rho=1 / 2$. The above properties resemble the properties of the large-amplitude periodic phase obtained in MF. At the same time they signal that precursors of the ordered phase (in this model present for $T^{*}=0$ ) have a strong effect on the mechanical ( $p$ and compressibility) and thermal $\left(c_{v}\right)$ properties.

On the other hand, the high- $T^{*}$ weakly ordered phase found in MF is only reflected in the qualitative change of properties of the correlation function. For $\mu^{*}$ that in the ground state corresponds to the gas or liquid, we observe that the correlation length assumes a maximum for some finite $T^{*}$, indicating increasing order for increasing $T^{*}$. Moreover, for such $T^{*}$ the amplitude of the correlation function changes from a very small value to a much larger value for a narrow range of $\mu^{*}$, and stays large for the range of $\mu^{*}$ similar to the stability region of the periodic phase found in MF.

The most surprising behavior shows the correlation function for $1 / 9<J^{*}<1 / 3$. It crosses over from a monotonic decay at low $T^{*}$ to an oscillatory decay for higher $T^{*}$ that is, however, much smaller than the temperature corresponding to the appearance of the periodic phase in MF. The unusual appearance of the periodic short-range order at intermediate temperatures that for weak repulsion is neither energetically nor entropically favored is the most surprising and interesting exact result of this work. It means that for competing interactions the inhomogeneities may appear as a compromise between the macroscopic phase separation that is energetically favorable but entropically unfavorable, and the disordered structure that is favorable entropically and unfavorable energetically.

\section{ACKNOWLEDGMENTS}

We thank E. Lomba and W. T. Góźdź for discussions. A part of this work was realized within the International Ph.D. Projects Programme of the Foundation for Polish Science, cofinanced from European Regional Development Fund within Innovative Economy Operational Programme "Grants for innovation." Partial support by the NCN Grant No. 2012/05/B/ST3/03302 is also acknowledged. N.G.A. gratefully acknowledges financial support from the Dirección General de Investigación Científica y Técnica under Grant No. FIS2010-15502, from the Dirección General de Universidades e Investigación de la Comunidad de Madrid under Grant No. S2009/ESP-1691 and Program MODELICO-CM.

\section{APPENDIX: TRICRITICAL POINT}

We assume that when the continuous transition to the homogeneous phase with density $\bar{\rho}_{0}\left(\mu^{*}, T^{*}\right)$ is approached, the density in the periodic phase has the form $\bar{\rho}(x)=\bar{\rho}_{0}+\Delta \rho(x)$ with

$$
\Delta \rho(x)=\delta \rho+\phi \cos \left(k_{b} x\right) .
$$

The continuous transition coincides with the $\lambda$-line, and at the $\lambda$-line $\delta \rho=\phi=0$. The difference between the grand potential in the periodic and the homogeneous phases, $\Delta \Omega=\Delta \omega L$, is a function of $\delta \rho$ and $\phi$ and $\Delta \omega$ can be Taylor expanded for $\delta \rho \rightarrow 0$ and $\phi \rightarrow 0$. When $2 \pi / k_{b}$ is not integer, in calculating $\Delta \omega$ (see (11)) we choose $n \rightarrow \infty$ such that $L \approx 2 n \pi / k_{b}$ and make the approximation:

$$
\frac{1}{L} \sum_{x=1}^{L} \cos \left(k_{b} x\right)^{m} \approx \frac{1}{2 \pi} \int_{0}^{2 \pi} \cos z^{m} d z .
$$

The second derivative of $\Delta \omega$ with respect to $\phi$ vanishes at the continuous transition, while the second derivative with respect to $\delta \rho$ is positive. From the extremum condition $\partial \Delta \omega / \partial \delta \rho=0$ we obtain

$$
\delta \rho=-\frac{A_{3}\left(\rho_{0}\right)}{4\left(\beta^{*} \tilde{V}(0)+A_{2}\left(\rho_{0}\right)\right)} \phi^{2}+O\left(\phi^{4}\right)
$$

and

$$
\beta \Delta \omega=a_{2} \phi^{2}+a_{4} \phi^{4}+O\left(\phi^{6}\right)
$$

where

$$
\begin{gathered}
a_{2}=\frac{\beta^{*} \tilde{V}\left(k_{b}\right)+A_{2}\left(\rho_{0}\right)}{4}, \\
a_{4}=\frac{1}{32}\left(\frac{A_{4}\left(\rho_{0}\right)}{2}-\frac{A_{3}\left(\rho_{0}\right)^{2}}{\beta^{*} \tilde{V}(0)+A_{2}\left(\rho_{0}\right)}\right),
\end{gathered}
$$


and $A_{n}(\rho)=d^{n} \beta f_{h}(\rho) / d \rho^{n}$ with $f_{h}$ given in Eq. (12). The transition is continuous for $a_{4}>0$, and becomes first order at the tricritical point (TCP) given by $a_{2}=a_{4}=0$. We obtain for the density and temperature at the TCP the following expressions:

$$
\begin{gathered}
\bar{\rho}_{0}^{t c p}=\frac{1}{2}\left[1 \pm \sqrt{\frac{\tilde{V}\left(k_{b}\right)-\tilde{V}(0)}{\tilde{V}\left(k_{b}\right)+3 \tilde{V}(0)}}\right], \\
T_{t c p}^{*}=\frac{-\tilde{V}\left(k_{b}\right) \tilde{V}(0)}{\tilde{V}\left(k_{b}\right)+3 \tilde{V}(0)} .
\end{gathered}
$$

Real positive solutions for $\bar{\rho}_{0}^{t c p}$ exist for $1 / 9<J^{*}<1$, i.e., when the lines of instability intersect and form a loop.

${ }^{1}$ J. N. Israelachvili, Intermolecular and Surface Forces, 3rd ed. (Academic Press, Boston, 2011).

${ }^{2}$ J. L. Barrat and J.-P. Hansen, Basic Concepts for Simple and Complex Liquids (Cambridge University Press, 2003).

${ }^{3}$ A. Stradner, H. Sedgwick, F. Cardinaux, W. Poon, S. Egelhaaf, and P. Schurtenberger, Nature (London) 432, 492 (2004).

${ }^{4}$ A. I. Campbell, V. J. Anderson, J. S. van Duijneveldt, and P. Bartlett, Phys. Rev. Lett. 94, 208301 (2005).

${ }^{5}$ M. Dijkstra, R. van Roij, and R. Evans, Phys. Rev. E 59, 5744 (1999).

${ }^{6}$ S. Buzzaccaro, J. Colombo, A. Parola, and R. Piazza, Phys. Rev. Lett. 105, 198301 (2010).

${ }^{7}$ A. Sánchez-Iglesias, M. Grzelczak, T. Altantzis, B. Goris, J. Perez-Juste, S. Bals, G. Van Tendeloo, S. H. Donaldson, Jr., B. F. Chmelka, J. N. Israelachvili, and L. M. Liz-Marzán, ACS Nano 6, 11059 (2012).

${ }^{8}$ A. Shukla, E. Mylonas, E. D. Cola, S. Finet, P. Timmins, T. Narayanan, and D. I. Sveergun, Proc. Natl. Acad. Sci. U.S.A. 105, 5075 (2008).

${ }^{9}$ S. L. Veatch, O. Soubias, S. L. Keller, and K. Gawrisch, Proc. Natl. Acad. Sci. U.S.A. 104, 17650 (2007).

${ }^{10}$ C. Hertlein, L. Helden, A. Gambassi, S. Dietrich, and C. Bechinger, Nature (London) 451, 172 (2008).

${ }^{11}$ A. Gambassi, A. Maciolek, C. Hertlein, U. Nellen, L. Helden, C. Bechinger, and S. Dietrich, Phys. Rev. E 80, 061143 (2009).

${ }^{12}$ B. B. Machta, S. L. Veatch, and J. P. Sethna, Phys. Rev. Lett. 109, 138101 (2012).

${ }^{13}$ R. P. Sear and W. M. Gelbart, J. Chem. Phys. 110, 4582 (1999).

${ }^{14}$ D. Pini, G. Jialin, A. Parola, and L. Reatto, Chem. Phys. Lett. 327, 209 (2000).

${ }^{15}$ A. Imperio and L. Reatto, J. Phys.: Condens. Matter 16, S3769 (2004).

${ }^{16}$ A. Imperio and L. Reatto, Phys. Rev. E 76, 040402 (2007).
${ }^{17}$ A. Imperio and L. Reatto, J. Chem. Phys. 124, 164712 (2006).

${ }^{18}$ D. Pini, A. Parola, and L. Reatto, J. Phys.: Condens. Matter 18, S2305 (2006).

${ }^{19}$ A. J. Archer, D. Pini, R. Evans, and L. Reatto, J. Chem. Phys. 126, 014104 (2007).

${ }^{20}$ A. Ciach, Phys. Rev. E 78, 061505 (2008).

${ }^{21}$ A. J. Archer, Phys. Rev. E 78, 031402 (2008).

${ }^{22}$ A. Archer, C. Ionescu, D. Pini, and L. Reatto, J. Phys.: Condens. Matter 20, 415106 (2008).

${ }^{23}$ A. J. Archer and N. B. Wilding, Phys. Rev. E 76, 031501 (2007).

${ }^{24}$ A. Ciach and W. T. Góźdź, Condens. Matter Phys. 13, 23603 (2010).

${ }^{25}$ D. F. Schwanzer and G. Kahl, J. Phys.: Condens. Matter 22, 415103 (2010).

${ }^{26}$ A. de Candia, E. DelGado, A. Fierro, N. Sator, M. Tarzia, and A. Coniglio, Phys. Rev. E 74, 010403(R) (2006).

${ }^{27}$ J. Toledano, F. Sciortino, and E. Zaccarelli, Soft Matter 5, 2390 (2009).

${ }^{28}$ A. Ciach and O. Patsahan, Condens. Matter Phys. 15, 23604 (2012).

${ }^{29}$ A. Ciach, Mol. Phys. 109, 1101 (2011).

${ }^{30}$ S. A. Brazovskii, Sov. Phys. JETP 41, 85 (1975).

${ }^{31}$ P. Bak, D. Mukamel, and S. Krinsky, Phys. Rev. Lett. 36, 52 (1976).

${ }^{32}$ R. Dickman and G. Stell, Phys. Rev. Lett. 77, 996 (1996).

${ }^{33}$ A. Ciach, J. Stat. Phys. 40, 593 (1985).

${ }^{34} \mathrm{M}$. Pretti and C. Buzano, J. Chem. Phys. 121, 11856 (2004).

${ }^{35}$ C. Buzano, E. D. Stefanis, and M. Pretti, J. Chem. Phys. 129, 024506 (2008).

${ }^{36}$ J. Roberts and P. G. Debenedetti, J. Chem. Phys. 105, 658 (1996).

${ }^{37}$ M. C. Barbosa, M. Deserno, C. Holm, and R. Messina, Phys. Rev. E 69, 051401 (2004).

${ }^{38}$ A. L. Balladares and M. C. Barbosa, J. Phys.: Condens. Matter 16, 8811 (2004).

${ }^{39}$ M. E. Fisher and W. Selke, Phys. Rev. Lett. 44, 1502 (1980).

${ }^{40} \mathrm{~W}$. Selke, Phase Transitions and Critical Phenomena (New York Academic, 1992), Vol. 15.

${ }^{41}$ W. Selke, Phys. Rep. 170, 213 (1988).

${ }^{42}$ A. Ciach, J. S. Høye, and G. Stell, J. Chem. Phys. 90, 1214 (1989).

${ }^{43}$ A. Ciach, J. Mol. Liq. 164, 74 (2011).

${ }^{44}$ A. Ciach, W. T. Góźdź, and R. Evans, J. Chem. Phys. 118, 3702 (2003).

${ }^{45}$ M. C. Barbosa, Phys. Rev. E 48, 1744 (1993).

${ }^{46}$ P. C. Royall, M. E. Leunissen, A.-P. Hynninen, M. Dijkstra, and A. van Blaaderen, J. Chem. Phys. 124, 244706 (2006).

${ }^{47}$ D. El. Masri, T. Vissers, S. Badaire, J. C. P. Stiefelhagen, H. R. Vutukuri, P. Helfferich, T. H. Zhang, W. K. Kegel, A. Imhof, and A. van Blaaderen, Soft Matter 8, 6979 (2012).

${ }^{48}$ D. Andelman, F. Brochard, and J.-F. Joanny, Proc. Natl. Acad. Sci. U.S.A. 84, 4717 (1987).

${ }^{49}$ D. Landau and K. Binder, A Guide to Monte Carlo Simulations in Statistical Physics, 2nd ed. (Cambridge University Press, 2005).

${ }^{50}$ N. G. Almarza, J. A. Capitán, J. A. Cuesta, and E. Lomba, J. Chem. Phys. 131, 124506 (2009). 